TO BE PUBLISHED IN THE ASTROPHYSICAL JOURNAL

Preprint typeset using $\mathrm{LT}_{\mathrm{E}} \mathrm{X}$ style emulateapj v. 12/01/06

\title{
CENTRAL STARS OF PLANETARY NEBULAE IN THE MAGELLANIC CLOUDS: A DETAILED SPECTROSCOPIC
} ANALYSIS*

\author{
J.E. HERALD, L. BIANCHI \\ Department of Physics and Astronomy, The Johns Hopkins University \\ To be published in the Astrophysical Journal
}

\begin{abstract}
We observed five central stars of planetary nebulae (CSPN) in the Large Magellanic Cloud (LMC) and three in the Small Magellanic Cloud (SMC) with the Far Ultraviolet Spectroscopic Explorer (FUSE), in the range $905-1187 \AA$. We performed a model-based analysis of these spectra in conjunction with Hubble Space Telescope (HST) spectra in the UV and optical range to determine stellar and nebular parameters. The signature of hot $(T \gtrsim 2000 \mathrm{~K})$ circumstellar molecular hydrogen is found in the FUSE spectra of most objects. We also find evidence of X-rays in the wind of LMC-SMP 76.

Subject headings: stars: AGB and Post-AGB - stars: atmospheres - stars: individual (LMC-SMP 1, LMCSMP 29, LMC-SMP 50, LMC-SMP 76, LMC-SMP 83, SMC-SMP 1, SMC-SMP 3, SMCSMP 5, SMC-SMP 22) - ultraviolet: stars
\end{abstract}

\section{INTRODUCTION}

Central Stars of Planetary Nebulae (CSPN) residing in the Magellanic Clouds (MCs) are an important asset to the study of CSPN evolution for two main reasons: their distances are well constrained, allowing their physical parameters (e.g., radius and luminosity) to be determined with much less uncertainty than their Galactic counterparts, and the metallicities of their host galaxies are lower than in the Milky Way. Metallicity is expected to influence a star's evolution by setting the efficiency of radiative driving during the phases when the star has a stellar wind: the asymptotic giant branch (AGB) phase, the post-AGB phase, and the [Wolf-Rayet] phase ([WR]). Higher metallicity, and thus wind radiative acceleration, should increase the star's mass-loss rate and wind velocity, altering the layers exposed and chemical yields as well as the kinematics of the nebular shell. This has implications for galaxy evolution, through chemical enrichment and the dynamic interactions between the star's ejected material and the surrounding interstellar medium (ISM). The large fraction of stars that pass through these phases make their contribution to the galactic chemical evolution significant (see, e.g., Marigo et al. 2001 for a discussion).

The far-UV wavelength range is useful for studying hot PN nuclei, as they emit the bulk of their observable flux in this range, and it contains many strong lines which are diagnostics of stellar parameters. It is also uncontaminated by nebular continuum emission (Bianchi et al. 1997; Herald \& Bianchi 2004a), which affects wavelengths longer than Ly $\alpha$, allowing us to characterize the physical parameters of both the nebular and central star components through detailed quantitative modeling.

Motivated by the above considerations, we (Herald \& Bianchi 2004a - hereafter, HB04) performed a spectroscopic analysis on far-UV and UV data of seven CSPN in the Large Magellanic Cloud (LMC) using data taken with the Far-Ultraviolet Spectroscopic Explorer (FUSE). As a continuation of that work, we have obtained FUSE observations of five additional LMC CSPN (Bianchi's D034

*BASED ON OBSERVATIONS MADE WITH THE NASA-CNES-CSA FAR ULTRAVIOLET SPECTROSCOPIC EXPLORER AND ARCHIVAL DATA. FUSE IS OPERATED FOR NASA BY THE JOHNS HOPKINS UNIVERSITY UNDER NASA CONTRACT NAS5-32985. program) and three Small Magellanic Cloud (SMC) CSPN (Bianchi's C056 program). These targets were selected from those expected to be the brightest (in the far-UV) from the photoionization studies of Dopita \& Meatheringham (1991a b); Dopita et al. (1997); Vassiliadis et al. (1996, 1998a). The additional LMC targets (D034) were selected to expand the temperature coverage of the HB04 LMC sample to higher temperatures, and with the SMC sample we gain objects in a much lower metallicity environment. Together with the LMC sample of Bianchi's FUSE proposal B001, analyzed by HB04, these programs cover all the known CSPN in the MC observable by FUSE. Our analysis is similar to that of $\mathrm{HB} 04$, and the reader is referred to that work for more details and background information.

This paper is arranged as follows. The observations and data reduction are described in $\S[2$ A comparison of the spectra of the objects is presented in $\$ 3$. Our models and parameter determinations are described in $\$ 4$ Individual object results are presented in $\S[5$. The implications of our results are discussed in $\S 6$ and our conclusions in $\$ 7$

\section{OBSERVATIONS AND REDUCTION}

For this work, we used far-UV spectra taken with FUSE and archive UV and optical spectra taken with the Hubble Space Telescope's (HST) Faint Object Spectrograph (FOS) or Space Telescope Imaging Spectrograph (STIS), which we now describe.

\subsection{Far-UV Spectra}

The FUSE observations (programs C056 and D034) of our sample stars are summarized in Table 1. They are some of the dimmest stellar objects yet observed by FUSE, and necessitated long integration times. FUSE covers the wavelength range $905-1187 \AA$ at a spectral resolution of $R \approx 20,000(\sim$ $15 \mathrm{~km} \mathrm{~s}^{-1}$ ). The instrument is described by Moos et al. (2000) and its on-orbit performance is discussed by Sahnow et al. (2000). FUSE collects light concurrently in four different channels (LiF1, LiF2, SiC1, and SiC2), each of which is divided into two segments (A \& B) recorded by two detectors, covering different subsets of the above range with some overlap. We used FUSE's LWRS $\left(30^{\prime \prime} \times 30^{\prime \prime}\right)$ aperture. These data, taken in "time-tag" mode, have been calibrated using 
the FUSE data reduction pipeline, efficiency curves and wavelength solutions (CALFUSE v2.4).

The spectra of these targets suffer from significant contamination from terrestrial airglow lines (such as $\mathrm{O}$ I and $\mathrm{N} \mathrm{I}$ ), and scattered solar emission features (O VI $\lambda \lambda 1032,38$ and C III $\lambda 1175)$, which often interfere with the spectroscopic analysis by obscuring the stellar features. For the LMC sample, we use "night-only" data (i.e., taken when the spacecraft was on the dark side of the Earth), which minimize this contamination, although some residual airglow lines (typically O I) can still appear (an example of the difference between day and night observations can be found in HB04).

Because of their faintness, most of our targets required many orbit-long exposures, each of which typically had low count-rates and thus signal-to-noise ratios. Each calibrated extracted sequence was checked for unacceptable count-rate variations (a sign of detector drift), incorrect extraction windows, and other anomalies. Segments with problems were not included in further steps. The default FUSE pipeline attempts to model the background measured off-target and subtracts it from the target spectrum. In some cases, the background is taken to be the model scattered-light scaled by the exposure time.

All the "good" calibrated segments/exposures were combined using FUSE pipeline routine and overlapping ranges of the different channels were then compared for consistency. There are several regions of overlap between the different channels, and these were used to ensure that the continuum matched. For the fainter targets, often the continuum levels for different detectors did not agree. The procedure we typically adopted was to trust the LiF1a absolute flux calibration (which is the most reliable - Sahnow et al. 2000), and scale the flux of the other detectors if needed. The LiF1b segment is sometimes affected by an artifact known as "the worm" (FUSE Data Handbook v1.3) and was omitted if the effect was found to be severe. The $\mathrm{SiC} 1$ detector seemed frequently offtarget, and other segments offered higher $\mathrm{S} / \mathrm{N}$ data in the same range, so its data was often not used. In the end, we typically used the $\mathrm{SiC} 2$ data for wavelengths below $\sim 1000 \AA$. Longwards of $1080 \AA$ LiF2a data was employed. For the intermediate region (i.e., 1000-1080 $\mathrm{A}$ ), data from LiF1b, LiF2b, or both were used (data from one segment was omitted if it was discrepant with that of the $\mathrm{SiC}$ detectors). The region between 1083 and $1087 \AA$ is not covered by the LiF detectors, and as the $\mathrm{SiC}$ detectors in this range were off-target, we have omitted this region (appearing as gaps in the plots). Table 1 shows which segments and portions were used for the combined spectrum. Data from near the detector edges were also omitted if they looked inconsistent.

Some individual objects warrant more discussion. The SMC observations had numerous difficulties. These observations occurred during a period of time after FUSE lost one of its reaction wheels, affecting its pointing, but before jitter correction had been introduced into the FUSE calibration pipeline to account for such effects. For SMC-SMP 1, it appears only the LiF1 was aligned with the central star with any reliability (the fine error sensor camera, used for guiding, is part of the LiF1 channel, so the location of the source is always aligned in the LiF1 apertures). Other detectors seemed to have gathered nebular emission lines with little or no stellar continuum for this object. We therefore only present the $\mathrm{LiF} 1$ data for this object but note that the LiF1b (longer wavelength) segment is often affected by "the worm" (see above).
Although the worm is not obviously present in the LiF1b observations of this object, the low count rate prevents a definitive statement regarding this. During the observation of SMCSMP 22, there was significant target drift as evidenced by inconstant count rates, and the $\mathrm{LiF} 2$ and $\mathrm{SiC} 2$ detectors seem to have been off-target. It also appears that another star was present in the aperture during much of the observation (discussed in $\S 5.2$. We present here data from the $\mathrm{SiC} 1$ and LiF1 detectors, but we stress that the absolute fluxes are uncertain due to what appears to be the target drifting in and out of the aperture throughout the observation. For SMC-SMP 5, no useful data was acquired. In the case of LMC-SMP 29, voltage and alignment problems resulted in no reliable FUSE data being collected.

The FUSE spectra presented in this paper were obtained by combining the good data from different segments, weighted by detector sensitivity, and rebinning to a uniform dispersion of $0.05 \AA$.

\subsection{UV and Optical Data}

Archival data from the HST's Faint Object Spectrograph (FOS) and Space Telescope Imaging Spectrograph (STIS) instruments were also used longwards of $1200 \AA$, as summarized in Table 2] The FOS archive data were taken in the $1^{\prime \prime}$ aperture, whose actual diameter following the COSTAR installation is $0.86^{\prime \prime}$. The angular sizes of the LMC PN are typically $\lesssim 1^{\prime \prime}$, so the FOS spectroscopy includes the entire nebula (the exception is LMC-SMP 83, see Table 3). The utilized FOS dispersers include G130H ( $\Delta \lambda \sim 1 \AA$ ), G190H $(\Delta \lambda \sim 1.5 \AA), \mathrm{G} 270 \mathrm{H}(\Delta \lambda \sim 2 \AA)$ ) and $\mathrm{G} 400 \mathrm{H}(\Delta \lambda \sim 3 \AA)$. For LMC-SMP 83, medium-resolution STIS data is available, taken through the $52^{\prime \prime} \mathrm{x} 0.2^{\prime \prime}$ aperture with the G140L $(\Delta \lambda \sim 1.3 \AA)$, G230L $(\Delta \lambda \sim 1.5 \AA)$, G430L $(\Delta \lambda \sim 1.6 \AA)$ and G750L $\Delta \lambda \sim 1.6 \AA$ ) gratings.

Generally, the flux levels of the FUSE and FOS/STIS data in the region of overlap are in good agreement for the LMC observations, except for LMC-SMP 83, which is known to be variable (\$5). The SMC sample contains more discrepancies, however. For SMC-SMP 22, the UV flux level is much higher than that of the far-UV (discussed more in $\$ 5.2$ ). For SMCSMP 3 and SMC-SMP 1, the FUSE flux levels are higher by about a factor of 2 and 1.5 (respectively) than the FOS data. International Ultraviolet Explorer (IUE) spectra of these objects display similar flux levels as those of FOS, therefore we we have scaled the FUSE flux levels to that of the FOS in these instances.

\section{DESCRIPTION OF THE SPECTRA}

The reduced FUSE spectra for the LMC sample, along with line identifications, are shown in Fig.11 and the corresponding UV spectra are shown in Fig. 2]

Wind features are definitely seen in the far-UV spectra of most stars: S VI $\lambda \lambda 933,44$, C III $\lambda 977$, and S VI $\lambda \lambda 933,44$ are visible in LMC-SMP 1 and LMC-SMP 76. The latter also shows C III $\lambda 1175$ and P V $\lambda \lambda 1118,28$. For LMC-SMP 50, the only feature obviously attributable to a stellar wind is O VI $\lambda \lambda 1032,38$. LMC-SMP 83 shows also nebular emission in $\mathrm{O}$ VI, implying a very hot central star (temperatures of $T_{\text {eff }} \gtrsim 125 \mathrm{kK}$ are needed to generate these lines through photoionization - Chu et al. 2004). In the far-UV spectra, $\mathrm{H}_{2}$ absorption makes the continuum level very difficult to set (\$4.1).

In the UV range, LMC-SMP 50 shows some nebular fea- 
tures and an underlying continuum, but strong stellar features are absent. LMC-SMP 83, LMC-SMP 1, and LMCSMP 76 all display a P-Cygni profile of $\mathrm{N} V \lambda \lambda 1238,43$, as well as C IV $\lambda \lambda 1548,51$ of different strengths. The blue edges of the P-Cygni profiles in both the far-UV and UV spectra indicate the terminal wind velocities of all objects are $\mathrm{v}_{\infty} \simeq 500-1000 \mathrm{~km} \mathrm{~s}^{-1}$, except in the case of LMC-SMP 83 $\left(\mathrm{v}_{\infty} \simeq 1600 \mathrm{~km} \mathrm{~s}^{-1}\right)$ and SMC-SMP $3\left(\mathrm{v}_{\infty} \geq 2000 \mathrm{~km} \mathrm{~s}^{-1}\right)$.

The reduced FUSE, and archive UV, spectra for the SMC sample are shown in Fig. 3 and Fig. 4 respectively. In the FUSE observations, nebular and airglow features contaminate the spectra (especially at short wavelengths). C III $\lambda 1175$ is present in absorption in the spectra of SMC-SMP 1 and SMCSMP 22. O VI $\lambda \lambda 1032,38$ nebular lines are also seen in the latter. The only wind features in the UV spectra are C IV $\lambda \lambda 1548,51$ seen in SMC-SMP 1 . SMC-SMP 22 shows a more highly-ionized nebular spectrum than that of LMC-SMP 83.

\section{MODELING}

Modeling the central stars of the MC CSPN presents some challenges. First, their nebulae are typically compact $\left(\lesssim 1^{\prime \prime}\right)$, and in most cases entirely contained in the FOS aperture used in the archival data available. Their UV and optical spectra are frequently contaminated by the nebular continuum and lines, which obscure and sometimes entirely mask the stellar spectrum in these regions. Thus, one must rely on a smaller set of stellar features in the far-UV and UV to determine the stellar parameters. Second, the far-UV region, while not influenced by nebular continuum emission, is affected by absorption by electronic transitions of sight-line molecular hydrogen $\left(\mathrm{H}_{2}\right)$. In our objects, such absorptions cover the entire far-UV region. The analysis of the spectra consists of modeling the central star spectrum, the nebular continuum emission longwards of $1200 \AA$, and the sight-line Hydrogen (atomic and molecular) shortwards concurrently and to find a consistent solution. However, we discuss each in turn for clarity. The reddening has to be derived concurrently, but it is small in all cases, and therefore it does not contribute much to the uncertainties.

Throughout this paper, we adopt a LMC distance of $D=$ $50.6 \mathrm{kpc}$ (Feast 1991), an SMC distance of $D=60.0 \mathrm{kpc}$ (Harries et al. 2003), an LMC metallicity of $z=0.4 Z_{\odot}$ (Dufour 1984), and an SMC metallicity of $z=0.1 \mathrm{Z}_{\odot}$, with values of "solar" abundances from Grevesse \& Sauval (1998).

As with $\mathrm{HB} 04$, we assume Galactic foreground extinctions of 0.05 (Bessell 1991), with the remainder being from the Magellanic Cloud. We use the Fitzpatrick (1999) reddening law for the LMC and the Cardelli et al. (1989) reddening law for the Galaxy $\left(R_{v}=3.1\right)$ extinction, extrapolated down to farUV wavelengths.

\subsection{Molecular and Atomic Hydrogen}

Absorption due to atomic (H I) and molecular hydrogen $\left(\mathrm{H}_{2}\right)$ along the sight-line often complicates the far-UV spectra of these objects. Toward a CSPN, this sight-line material typically consists of interstellar and circumstellar components (e.g., Herald \& Bianchi 2002, 2004a b). Material comprising the circumstellar $\mathrm{H}$ I and $\mathrm{H}_{2}$ presumably was ejected from the star earlier in its history, and is thus important from an evolutionary perspective.

The $\mathrm{H}_{2}$ transitions in the FUSE range are from the Lyman $\left(B^{1} \Sigma_{u}^{+}-X^{1} \Sigma_{g}^{+}\right)$and Werner $\left(C^{1} \Pi_{u}^{ \pm}-X^{1} \Sigma_{g}^{+}\right)$series. We (HB04) found that, for our previous LMC CSPN, a significant amount of hot (i.e., $T \gtrsim 2000 \mathrm{~K}) \mathrm{H}_{2}$ lies along the sight-lines, presumably associated with the nebula. At such temperatures, higher vibrational states become populated and the absorption pattern is much more complex, obscuring the entire far-UV continuum spectrum (see Fig. 5 of HB04). With care, the effects of such a gas can be accounted for and strong stellar features can be discerned, but weaker stellar features will be unrecoverable.

We have modeled the $\mathrm{H}_{2}$ toward our sample CSPN in the following manner. For a given column density $(N)$ and gas temperature $(T)$, the absorption profile of each line is calculated by multiplying the line core optical depth $\left(\tau_{0}\right)$ by the Voigt profile $[H(a, x)]$ (normalized to unity) where $x$ is the frequency in Doppler units and $a$ is the ratio of the line damping constant to the Doppler width (the "b" parameter). The observed flux is then $F_{o b s}=\exp \left[-\tau_{0} H(a, x)\right] \times F_{\text {intrinsic }}$. We first assume the presence of an interstellar component with $T=80 \mathrm{~K}$ (corresponding to the mean temperature of the ISM - Hughes et al. 1971) and $v_{\text {turb }}=10 \mathrm{~km} \mathrm{~s}^{-1}$. The column density of this interstellar component is estimated by fitting its strongest transitions. If additional absorption features due to higher-energy $\mathrm{H}_{2}$ transitions are observed, a second (hotter) component is added, presumed to be associated with the nebula. We thus velocity-shift the $\mathrm{H}_{2}$ absorption features of the circumstellar component to the radial velocity (from Table 3) of the particular star. HB04 found that, typically, the absorption pattern of the circumstellar hot $\mathrm{H}_{2}$ was adequately fit by absorption models with $T_{\text {eff }} \simeq 2000 \mathrm{~K}$ and $\log N \simeq 16.7 \mathrm{~cm}^{-2}$, and the reader is referred to that work for a more detailed discussion of the $\mathrm{H}_{2}$ fitting. We note that our terminology of "circumstellar" and "interstellar" components is a simplification, indicating that we assume the "cool" component to be mostly interstellar and the "hot" component mostly circumstellar. However, the column density derived for the cooler "interstellar" component may also include circumstellar $\mathrm{H}_{2}$. Our derived parameters for sight-line Hydrogen are shown in Table 5 Note for some objects (e.g., LMCSMP 29 and SMC-SMP 1), we have not attempted to fit the $\mathrm{H}_{2}$ spectrum, due to the lack of useful FUSE data. If a number appears in the table without uncertainties, that value has been assumed rather than derived. Molecular hydrogen disassociates around $T \simeq 2500$, so such high temperatures are signs of non-equilibrium conditions (note our models assume thermal populations). Such $\mathrm{H}_{2}$ characteristics are observed in shocked regions. A shocked region is likely complex, containing areas of $\mathrm{H}_{2}$ of differing characteristics. Because of the high complexity of the absorption pattern at these hot temperatures, we did not attempt to fit every feature, but the absorption pattern in the far-UV as a whole, to a point where the stellar features could be identified and modeled.

When possible, H I column densities are determined from the $\operatorname{Ly} \alpha$ and $\operatorname{Ly} \beta$ features, which encompass both the interstellar and possible nebular components. The absorption profiles of $\mathrm{HI}$ are calculated in a similar fashion as described above for $\mathrm{H}_{2}$.

We also list in Table 5 the reddenings implied by our measured column densities of $\mathrm{HI}$ using the relationship $\left\langle N(\mathrm{H} \mathrm{I}) / E_{\mathrm{B}-\mathrm{V}}\right\rangle=4.8 \times 10^{21} \mathrm{~cm}^{-2} \mathrm{mag}^{-1}$ (Bohlin et al. 1978), which represents typical conditions in the (Galactic) ISM.

\subsection{The Nebular Continuum}

Nebular parameters taken from the literature for the program objects are compiled in Table 3 They include the angular size of the nebula $\theta$, the nebular radius $r_{n e b}$, the expansion 
velocity $v_{\exp }$, the electron density $n_{e}$, the electron temperature $T_{e}$, the $\mathrm{H} \beta$ flux $F_{H \beta}$, the Helium to Hydrogen ratio and the doubly to singly ionized Helium ratio. Values in bold are our derived values (see below). We also list the reddenings determined from literature values of the logarithmic extinction at $\mathrm{H} \beta$ using the relation $c_{H \beta}=1.475 E_{\mathrm{B}-\mathrm{V}}$.

In some cases, the nebular continuum significantly contributes to the observed spectra at wavelengths $\gtrsim 1200 \AA$ and must be estimated to fit the data $(\$ 4.3)$.

The nebular continuum emission has been modeled using the code described in Bianchi et al. (1997), which accounts for two-photon, $\mathrm{H}$ and $\mathrm{He}$ recombination, and free-free emission processes. The computed emissivity coefficient of the nebular gas is scaled as an initial approximation to the total flux at the Earth by deriving the emitting volume from the dereddened absolute $\mathrm{H} \beta$ flux. In the case of LMC-SMP 83, the nebula was not entirely contained within the aperture used for the STIS observations, and so we scaled the continuum by an appropriate geometrical factor $\left(4 A / \pi \theta^{2}\right.$ where $A$ is the area of the aperture in square arcseconds). $T_{e}$ and $n_{e}$ from the literature are used as initial inputs, and adjusted if necessary. LMC-SMP 1 and LMC-SMP 76 have no published values of the $\mathrm{He}^{2+} / \mathrm{He}^{+}$ratio. For these, we found adopting a ratio of 0 to produce satisfactory fits (these are relativity cooler objects). The values of $F_{H \beta}$ and $\mathrm{c}_{H \beta}$ are perhaps the most uncertain, and we finally scale the nebular flux with the assumption that it is responsible for the majority of the flux at longer wavelengths. The nebular continuum flux level is further constrained not to exceed the P-Cygni troughs in the UV range. Our combined stellar and nebular models, along with the observations, are shown in Fig. 5

\subsection{The Central Stars}

To model the spectra of the central stars, we used CMFGEN (Hillier \& Miller 1998, 1999; Hillier et al. 2003), a line-blanketed code suitable for an extended, sphericallysymmetric expanding atmosphere. We also used the hydrostatic structures from TLUSTY (Hubeny \& Lanz 1995) models as input to the CMFGEN models. TLUSTY calculates the atmospheric structure assuming a plane-parallel geometry. The detailed workings of the code are explained in the references above, here we give a brief descriptions of the salient features.

For CMFGEN models, the fundamental photospheric/wind parameters include $T_{e f f}, R_{*}$, the mass loss rate $\dot{M}$, the elemental abundances, the velocity law and the wind terminal velocity, $\mathrm{v}_{\infty} . R_{*}$ is taken to be the inner boundary of the model atmosphere (corresponding to a Rosseland optical depth of $\sim 20$ ). The stellar temperature $T_{*}$ is related to the luminosity and radius by $L=4 \pi R_{*}{ }^{2} \sigma T_{*}{ }^{4}$, and the effective temperature $\left(T_{e f f}\right)$ is similarly defined but at a radius corresponding to a Rosseland optical depth of $2 / 3\left(R_{2 / 3}\right)$. The luminosity is conserved at all depths, so $L=4 \pi R_{2 / 3}^{2} \sigma T_{\text {eff }}{ }^{4}$.

We assumed what is essentially a standard velocity law for the stellar wind $v(r)=\mathrm{v}_{\infty}\left(1-r_{0} / r\right)^{\beta}$ where $r_{0}$ is roughly equal to $R_{*}$. The choice of velocity law mainly affects the profile shape, not the total optical depth of the wind lines, and does not greatly influence the derived stellar parameters. Once a velocity law is specified, the density structure of the wind $\rho(r)$ is then parameterized by the mass-loss rate $\dot{M}$ through the equation of continuity: $\dot{M}=4 \pi R_{*}{ }^{2} \rho(r) v(r)$. However, one actually can only derive $\dot{M}_{s m}=\left(\dot{M}_{c l} / \sqrt{f}\right)$ from the models, where $\dot{M}_{s m}$ and $\dot{M}_{c l}$ are the smooth and clumped mass- loss rates, and the degree of clumpiness is parametrized by the filling factor $(f)$. Both theory (Owocki et al. 1988, 1994) and observations indicate that radiation driven winds are clumped to some extent. Unless otherwise noted, $\dot{M}$ refers to $\dot{M}_{s m}$ throughout this paper.

It has been found that wind models with the same transformed radius $R_{t}\left[\propto R_{*}\left(\mathrm{v}_{\infty} / \dot{M}\right)^{2 / 3}\right]$ (Schmutz et al. 1989) and $\mathrm{v}_{\infty}$ have the same ionization structure, temperature stratification and spectra (aside from appropriate scalings with $R_{*}$ - Schmutz et al. 1989; Hamann et al. 1993). Thus, once the velocity law and abundances are set, one parameter may be fixed (say $R_{*}$ ) and parameter space can then be explored by varying only the other two parameters (e.g., $\dot{M}$ and $\left.T_{\text {eff }}\right)$. For opacities which are proportional to the square of the density, the optical depth of the wind scales as $\propto R_{t}^{-3}$, so $R_{t}$ can be thought of as an optical depth parameter.

In the CMFGEN models presented by HB04, a constant scale height $(h)$ was adopted, which connects the spherically extended hydrostatic outer layers to the wind. The gravity is related to the scale height as $h \propto g^{-1}$. Our models here differ in this respect, as instead of adopting a constant scale height, we have used the hydrostatic structures as generated from our TLUSTY models in the CMFGEN models, in the manner as described by Bouret et al. (2003). Because of the severe $\mathrm{H}_{2}$ absorption in the far-UV, and the masking of the stellar flux by the nebular continuum at longer wavelengths, the gravity cannot be well constrained in our analysis. Thus, we adopt gravities typical of CSPNe of the given $T_{e f f}$, guided by the $\log g-T_{\text {eff }}$ relations of Vassiliadis \& Wood (1994) (hereafter, VW94) (e.g., $\log g=4.7$ for $T_{\text {eff }} \simeq 55 \mathrm{kK}, \log g=6.0$ for $T_{\text {eff }} \simeq 100 \mathrm{kK}$, etc.). The wind features (used as diagnostics) are not very sensitive to the gravity, and typically the observed wind features can be fit with models of the same $T_{\text {eff }}$ but with gravities differing by over a magnitude. Line profiles in the optical, which would provide good diagnostics of the gravity, are not available for our sample (the G750L STIS data of LMC-SMP 83 does cover the optical range, but due to its emission line spectrum, provides no useful gravity diagnostics).

\subsubsection{Abundances and Model Ions}

We note here that throughout this work, the nomenclature $X_{i}$ represents the mass fraction of element $i$, and ' $\mathrm{X}_{\odot}$ " denotes the solar value. Following HB04, we adopted two model abundance patterns for the MC CSPN. The first, appropriate for "H-rich" CSPN, have solar abundances for $\mathrm{H}$ and He but assume LMC/SMC metallicities of $z=0.4 / 0.1 \mathrm{Z}_{\odot}$ for the metals. For the second, corresponding to "H-deficient" (or H-poor) CSPN, we have assumed no hydrogen and an abundance pattern of $X_{H e} / X_{C} / X_{N} / X_{O}=0.55 / 0.37 / 0.01 / 0.08$ with LMC/SMC metallicities of $z=0.4 / 0.1 \mathrm{Z}_{\odot}$ for the other elements (e.g., S, Si, \& Fe). The abundances for the H-rich and $\mathrm{H}$-deficient grids are summarized in Table 4. H-deficient abundances (which are enriched in oxygen and carbon) are signs of He-burning. Normal, "H-rich" abundances can be seen when the star is either $\mathrm{H}$ or He-burning.

We mention here that there are no diagnostics in the far-UV or UV spectra of our objects that allow us to make a statement regarding their hydrogen/helium ratio. The handful of wind lines upon which we are basing our parameter determinations do not allow us to make firm statements regarding the specific abundances of a given element, but in most cases we are able to determine which grid is more appropriate based on 
the strength of carbon and oxygen features.

For the model ions, both TLUSTY and CMFGEN utilize the concept of "superlevels", whereby levels of similar energies are grouped together and treated as a single level in the rate equations (after Anderson 1989). Ions and the number of levels and superlevels included in the model calculations, as well references to the atomic data, are given in the Appendix $(\S \mathrm{A})$.

\subsubsection{Diagnostics}

Our sample covers a wide range of temperatures and massloss rates. As different diagnostics are used in different parameter regimes, we shall discuss the diagnostics used in a case-by-case basis $(\$ 5)$ and here we discuss only the more general diagnostics.

For stars showing strong wind features in their spectra, the terminal wind velocity $\left(\mathrm{v}_{\infty}\right)$ can be estimated from the blue edge of the P-Cygni absorption features. Strong P-Cygni profiles unobscured by $\mathrm{H}_{2}$ absorption or nebular emission are rare in the far-UV spectra of our sample. We mostly used C IV $\lambda \lambda 1548-51$ (when present) for our initial estimate of $\mathrm{v}_{\infty}$, and adjusted this value to match the wind lines. Due to lack of suitable diagnostics, we did not attempt to rigorously constrain the degree of clumping in the winds of our sample. In the parameter regime of our objects, the model spectra are mainly insensitive to $f$, except for O VI $\lambda \lambda 1032,38$ in the hotter models ( $T_{\text {eff }} \gtrsim 50 \mathrm{kK}$ ) and $\mathrm{P} \mathrm{V} \lambda \lambda 1118,28$ for cooler models (HB04). We note here that the smooth mass-loss rate $\dot{M}_{s m}$ is an upper limit of the actual mass-loss rate.

For the other parameters, we adopted the following method. We compared the observed spectra with the theoretical spectra of our model grids to determine the stellar parameters $T_{\text {eff }}$ and $R_{t}$ (in the case of CMFGEN models) or $T_{e f f}$ and $\log g$ (in the cast of TLUSTY models). Far-UV and UV flux levels (corrected for the nebular contribution) were used to set $R_{*}$ (since the distance is known), and thus $\dot{M}$ and $L$ can be derived from $R_{t}$. Uncertainties in the quoted parameters reflect the range of acceptable model fits.

Typically, one determines $T_{e f f}$ by the ionization of the CNO elements. For these objects, we typically use C III/C IV and/or O IV/O V/O VI to constrain $T_{\text {eff }}$. It is desirable to use diagnostics from many elements to ensure consistency. The presence or absence of some features offer further constraints. For example, for mass-loss rates in the regime of our stars, Si IV $\lambda \lambda 1394,1402$ disappears at temperatures $T_{\text {eff }} \gtrsim 50 \mathrm{kK}$, $\mathrm{S} \mathrm{IV} \lambda \lambda 1062,1075$ at $\sim 45 \mathrm{kK}$, and P V $\lambda \lambda 1118,28$ at $\sim 60 \mathrm{kK}$. We generally use all wind lines to constrain $\dot{M}$.

\section{STELLAR MODELING RESULTS}

Our derived central star parameters are summarized in Table 6 . We also indicate the model abundances used for each object, and list the reddenings determined by fitting the multiple components to the far-UV/UV spectra. We now discuss the modeling diagnostics and results for the individual objects.

\subsection{Results for LMC CSPN}

LMC-SMP 76 (Fig. 6)

The far-UV and UV spectra of this object are rich in wind features: S VI $\lambda \lambda 933,44, \mathrm{C}$ III $\lambda 977 \& 1175$, P V $\lambda \lambda 1118,28$, $\mathrm{O}$ VI $\lambda \lambda 1032,38, \mathrm{~N}$ V $\lambda \lambda 1238,43$, O V $\lambda 1371$, and C IV $\lambda \lambda 1548,51$. The O VI doublet has particularly strong absorption (but some of the emission seen includes $\mathrm{O}$ I airglow lines).
The strong carbon and oxygen spectrum exhibited by this object are best fit by models from our H-deficient grid (implying He-burning). In the model shown $\left(T_{e f f}=50 \mathrm{kK}\right.$, $\dot{M}=3.15 \times 10^{-8} \quad \mathrm{M}_{\odot} \mathrm{yr}^{-1}$ ) most of the features mentioned above are fit adequately, except $\mathrm{P}$ V $\lambda \lambda 1118,28$ (which is weak) and C III $\lambda 1175$ (which is strong). Adjusting the temperature/mass-loss rate within the quoted uncertainties alleviate these problems, at the expense of degrading the fits of some of the other diagnostics. In cooler models the C III features becomes too strong, unobserved Si IV $\lambda \lambda 1394,1402$ appears, and $\mathrm{N}$ V $\lambda \lambda$ 1238-43 disappears. Hotter models ( $T_{\text {eff }} \gtrsim 60 \mathrm{kK}$ ) lose the C III lines. Models fail to duplicate the strong O VI line in any case, which requires $T_{\text {eff }} \gtrsim 70 \mathrm{kK}$ before it begins to be fit adequately. This problem is alleviated by including X-rays in the model atmosphere (discussed below). Based on the C III and C IV wind lines, we derive a terminal wind velocity of $\mathrm{v}_{\infty}=1250 \pm 250 \mathrm{~km} \mathrm{~s}^{-1}$.

Our parameters for this star fall between the $\left(M_{i}, M_{f}\right)=$ $(1.0,0.578) \mathrm{M}_{\odot}$ and $(1.5,0.626)$ (He-burning) tracks $(z=$ 0.008 ) of VW94. This, combined with a better fit of the observation with models from our $\mathrm{H}$-deficient (i.e., $\mathrm{C}$ and $\mathrm{O}$ enriched) grid, cause us to conclude LMC-SMP 76 to be a He-burner. This is in contrast to Dopita et al. (1997), who concluded this object to be H-burning from comparison with evolutionary tracks based on their higher derived luminosity of $\log L=3.735 \mathrm{~L}_{\odot}$. They also inferred a very high nebular carbon abundance for this object, which is consistent with our findings that the wind is carbon-enriched. Bianchi et al. (1997) determined $T_{\text {eff }}=52 \mathrm{kK}, \mathrm{v}_{\infty}=1500 \mathrm{~km} \mathrm{~s}^{-1}$ for this object by modeling its UV and optical spectra, basically confirmed by our analysis.

The FUSE spectra of this star show the signature of hot molecular hydrogen, which severely attenuates the stellar flux. We have fit the absorption pattern with circumstellar $\mathrm{H}_{2}$ component having a temperature of $T \simeq 3000 \mathrm{~K}$.

As mentioned above, our models failed to fit the $\mathrm{O}$ VI $\lambda \lambda 1032,38$ line simultaneously with the other, lowerionization diagnostics. It is known that X-rays, arising from shocks believed to develop from instabilities in the radiationally-driven wind, are needed to explain observed $\mathrm{O}$ VI line profiles in $\mathrm{O}$ and early B-stars (Bianchi \& Garcia 2002; Garcia \& Bianchi 2004; Bianchi et al. 2006). We thus calculated some models which include X-rays in the wind. CMFGEN implements X-rays as described in Martins et al. (2005). In summary, shock sources are assumed to be distributed throughout the atmosphere once the wind achieves a certain velocity, with emissivities taken from Raymond \& Smith (1977). The shock parameters include the shock temperature (which controls the energy distribution of the shocks), the volume filling factor (which sets the level of emission), and the velocity of the wind where the shocks are switched on. For the starting velocity, we have used $\sim 400 \mathrm{~km} \mathrm{~s}^{-1}$, as radiative shocks are believed to become important when the wind achieves this velocity. We adopted a shock temperature of $300 \mathrm{kK}$, typical of high energy photons in O type stars (Schulz et al. 2003; Cohen et al. 2003), and adjusted the volume filling factor until adequate fits of the $\mathrm{O}$ VI feature were achieved. CMFGEN outputs the total X-ray luminosity, and the X-ray luminosities in the energy range above 1 and $0.1 \mathrm{keV}$, for the fluxes both absorbed by the wind, the X-ray flux which escapes. For our adopted parameters, the X-ray luminosity above $0.1 \mathrm{keV}$ is about 5 times that above $1 \mathrm{keV}$. We found that for $\log \mathrm{L}_{\mathrm{x}} / \mathrm{L}_{\mathrm{bol}} \gtrsim-9.0$, 
the strength of the absorption and the red emission components of the O VI P-Cygni profile were adequately duplicated (see example Fig. 7), although the blue emission component is too strong (most probably due to our simple velocity law). For greater $\mathrm{L}_{\mathrm{x}}$, the emission feature was a bit stronger than observed. Other spectral features did not change until $\log \mathrm{L}_{\mathrm{x}} / \mathrm{L}_{\mathrm{bol}} \simeq-6.0$, at which point $\mathrm{N}$ IV $\lambda 955$ decreased in strength. The $\mathrm{O}$ VI feature did not change with respect to our model without X-rays for $\log \mathrm{L}_{\mathrm{x}} / \mathrm{L}_{\text {bol }} \lesssim-10.0$. Note that these X-ray luminosities represent the escaped X-ray flux, i.e., what would be observed. The actual X-ray energy input into the wind is $\sim 500$ times more. Guerrero et al. (2001) discuss the possibility that shocks in the stellar wind may explain the observed, point-source X-ray fluxes $\left(\log \mathrm{L}_{\mathrm{x}} / \mathrm{L}_{\mathrm{bol}} \lesssim-7.0\right)$ of the central star of the Cat's Eye nebula (NGC 6453).

LMC-SMP 1 (Fig. 8)

The spectrum of LMC-SMP 1 is similar to that of LMCSMP 76, but with the wind features having a lower terminal velocity $\left(\mathrm{v}_{\infty} \lesssim 800 \mathrm{~km} \mathrm{~s}^{-1}\right)$ and weaker oxygen signature. We can achieve adequate fits using either models from the $\mathrm{H}$-rich or H-poor grid, with the former requiring higher massloss rates. We list both models in Table 6 but only show the $\mathrm{H}$-poor model in the figures. The higher temperature limit $\left(T_{e f f}=70 \mathrm{kK}\right)$ fits the O VI $\lambda \lambda 1032,38$ absorption trough better. For the lower temperatures $\left(T_{\text {eff }}=55 \mathrm{kK}\right)$ and mass-loss rates, the $\mathrm{C}$ III $\lambda 1175$ feature is too strong compared with the observations. In the $\mathrm{UV}$, the $\mathrm{N} \mathrm{V} \lambda \lambda 1238,43$ and $\mathrm{C} \mathrm{IV}$ $\lambda \lambda 1548,51$ are adequately fit with models in the quoted parameter range, but the $\mathrm{OV} \lambda 1371$ feature is always too strong (HB04 had similar problems with this line with some of their sample) in the case of either abundance pattern. The O IV $\lambda \lambda 1339,43$ feature is similarly too strong in the H-poor model spectra, while the carbon features are generally better-fit than with our H-rich model grid (probably indicating our adopted oxygen abundance is too high). The FUSE spectrum shows the absorption pattern from hot $(T \simeq 3000 \mathrm{~K})$ molecular hydrogen, which severely depresses the stellar flux. In comparison with LMC-SMP 76, this object has a higher luminosity and effective temperature, but a lower $\mathrm{v}_{\infty}$ (mass-loss rates are comparable). This could be explained by our finding that the wind of LMC-SMP 76 seems more obviously chemically enriched, and thus a higher opacity leading to a more efficient transfer of radiation-to-wind momentum.

LMC-SMP 83 (Fig. 9):

LMC-SMP 83 is a bit of an oddball, for which the classification as a CSPN is a matter of current debate. The object is notable for multiple reasons. Between 1993 and 1994, its luminosity increased from $\log L=4.6$ to $5.6\left(\mathrm{~L}_{\odot}\right)$. The quiescent luminosity is already extreme for a CSPN (most LMC CSPN have $\log L \lesssim 3.9 \mathrm{~L}_{\odot}$, e.g., Dopita \& Meatheringham $1991 \mathrm{a}$ b), but is less luminous than any known massive WN (e.g., Hamann \& Koesterke 1998). Furthermore, it is unique in that it displays a [WN] spectrum (other LMC CSPN which have WR-type spectra show [WC] spectra). It has been the subject of a long-term monitoring campaign and UV spectra exist spanning many epochs. Hamann et al. (2003) (hereafter, HPGR) performed detailed UV and optical spectral modeling across these epochs, and found the following parameters to be constant: $T_{*}=112 \pm 20 \mathrm{kK}, X_{H}=0.2, X_{N}=3 \times 10^{-3}$, $X_{C} \leq 1 \times 10^{-4}, X_{F e}=2 \times 10^{-4}$. The increase in luminosity during outburst was apparently caused by an increase in massloss rate, which increased the effective radius. The nature of LMC-SMP 83 is debatable, and HPGR discuss many alternatives to the CSPN classification (e.g., single massive star, low-mass binary system with mass transfer, double degenerate merger). The main argument against the CSPN classification is its surface nitrogen-rich abundances, which are difficult to explain in the CSPN context.

In Fig. 10, we show the absorption pattern of the interstellar hydrogen absorption component, and an additional hot circumstellar component applied to a flat continuum. There does appear to be an overall signature of hot $\mathrm{H}_{2}$ in the data, although many minor features are not well fit. The $\mathrm{H}_{2}$ absorptions are significant, and no obvious strong stellar features are apparent in the FUSE observations. Because the FUSE range offers no further stellar diagnostics, we have not performed much further modeling of this object beyond that of HPGR.

The FUSE flux levels are a factor of about 1.5 higher compared to the STIS data. LMC-SMP 83 was observed by STIS in 1999 and again in 2000, and comparison of these two data sets shows the 1999 continuum level in the 1250-1550 $\AA$ region to be about $95 \%$ that of the 2000 level measured 13 months later (the FUSE data was gathered about $1.5 \mathrm{yr}$ after the 2000 STIS observations). As noted by Hamann et al. (2003), there is very little change in the actual spectral features within this time. For this work we have used the 1999 STIS data set, because its signal to noise is superior to that of the 2000 data set, and scaled the FUSE data to the STIS flux levels.

We first computed a model with parameters similar to what HPGR found for the most recent epoch (2000): $\mathrm{v}_{\infty}=$ $1600 \mathrm{~km} \mathrm{~s}^{-1}, R_{*}=0.5 \mathrm{R}_{\odot}, T_{\text {eff }}=95 \mathrm{kK}, \log \dot{M}=-5.67 \mathrm{M}_{\odot}$, and the constant parameters quoted above (abundances and $T_{*}$ ). However, rather than using their reddening (a Galactic foreground reddening of $E_{\mathrm{B}-\mathrm{V}}=0.03 \mathrm{mag}$ and an LMC reddening of $E_{\mathrm{B}-\mathrm{V}}=0.13 \mathrm{mag}$ ), we used a smaller amount of reddening, to produce better agreement over our expanded wavelength range. The use of this less severe reddening results in a slightly smaller derived radius (and corresponding massloss rate) than those of HPGR. This model produced several He II features in the FUV which do not appear to be present in the observations. However, it is possible that severe absorption from a hot circumstellar $\mathrm{H}_{2}$ gas $(\$ 4.1)$ could attenuate the He II features to agree with the observations, as shown in Fig.9. Another possibility is that the mass-loss rate has since declined to the point these features no longer appear in emission. Note that because we have essentially adopted the stellar parameters of this object from HPGR and not performed any further stellar modeling, we have omitted uncertainties for its stellar parameters in Table 6 .

LMC-SMP 50 (Fig. 11)

The photoionization temperature for this star is $100 \mathrm{kK}$ (see Table 7), and we find that a H-rich model with $T_{\text {eff }} \simeq 110 \mathrm{kK}$, $\log g \simeq 6.0, \log \dot{M}=-8.2 \mathrm{M}_{\odot}, \mathrm{v}_{\infty} \simeq 500 \mathrm{~km} \mathrm{~s}^{-1}, R_{*} \simeq$ $0.21 \mathrm{R}_{\odot}$ does an adequate job at fitting the most apparent wind feature displayed - that of the $\mathrm{O}$ VI doublet (see Fig. 1). These parameters lie slightly below the $M_{\text {init }}=2.0 \mathrm{M}_{\odot}, M=$ $0.67 \mathrm{M}_{\odot}$ track of VW94. The rest of the theoretical spectrum is relatively featureless, however, whereas the FUSE and FOS display some complexity. Many of the absorption features in the shorter wavelength region of the FUSE spectrum can be attributed to molecular hydrogen, as shown in the figure. The poor quality of the observations make firm identification of stellar and interstellar features difficult. For example, the FOS spectra show a P-Cygni-like feature at $\lambda 1335$, which might be a C II feature of stellar origin (indicating a much cooler object, possibly a companion) or possibly a circumstellar absorption 
feature in a relatively noisy spectrum that mimics an actual PCygni line. The lack of strong, clean stellar features prohibit a more rigorous determination of stellar parameters.

LMC-SMP 29

Along with SMC-SMP 22, the FOS spectra show show high-ionization lines (e.g., $\mathrm{N} V \lambda \lambda 1238,43)$ and have extreme photoionization temperatures $(T \simeq 200 \mathrm{kK})$. Unfortunately, problems during the FUSE observation ( $\$ 2$ resulted in unreliable FUSE data for this object, which we have not attempted to model. We have also made no attempt to fit the interstellar Ly $\alpha$ absorption feature.

\subsection{Results for SMC CSPN}

\section{SMC-SMP 3 (Fig. 12)}

There are no obvious wind features in the FOS spectrum of this object, and the FUSE spectrum only obviously shows the $\mathrm{O}$ VI resonance line. The FUSE spectrum is also contaminated by several strong airglow features, especially at shorter ( $\lesssim 1000 \AA$ ) wavelengths, and it shows a moderate $\mathrm{H}_{2}$ signature, which we have fitted. This star has a relatively high photoionization temperature of $T_{\text {eff }}=92 \mathrm{kK}$ (Table 7). Given the paucity of wind diagnostics, we can only speak in general terms about its parameters. Models with $90 \leq T_{\text {eff }} \leq 100 \mathrm{kK}$ with $-8.0<\log \dot{M}<-7.0 \mathrm{M}_{\odot}$ and $\mathrm{v}_{\infty} \gtrsim 2000 \mathrm{~km} \mathrm{~s}^{-1}$ adequately reproduce the $\mathrm{O}$ VI feature. Cooler temperatures and higher mass-loss rates typically show unobserved spectral features, and lower mass-loss rates result in a O VI weak compared to observations. Lower $\mathrm{v}_{\infty}$ results in both components of the O VI doublet being resolved - contrary to observations. However, the P-Cygni edge of the O VI feature is obscured by absorption due to $\mathrm{Ly} \beta$, so we cannot make a more precise measurement of $\mathrm{v}_{\infty}$. We conclude by saying a $\mathrm{H}$-rich model with temperatures consistent with the photo-ionization temperature with $\dot{M}$ and $\mathrm{v}_{\infty}$ within the previously stated limits fit the FUSE and FOS spectra adequately, with the caveat that these parameters are constrained mainly by the O VI doublet and the absence of other strong features. For comparison with evolutionary models, a $T_{\text {eff }}=95 \mathrm{kK}$ model scaled to the observed FOS flux levels would have $R_{*} \simeq 0.32 \mathrm{R}_{\odot}, \log L \simeq$ $3.8 \mathrm{~L}_{\odot}$. Comparison with the $Z=0.004, M_{\text {init }}=1.5 \mathrm{M}_{\odot}$, $M=0.640 \mathrm{M}_{\odot}$ track of VW94 implies a post-AGB age of a few kyr.

For this object, as well as LMC-SMP 50, we have relied mainly on the $\mathrm{O}$ VI doublet as a diagnostic. In our discussion of LMC-SMP 76, we showed this line could be heavily influenced by X-rays, which might add additional uncertainty to parameters derived using this line. However, photoionization models indicate that SMC-SMP 3 (and LMC-SMP 50) are considerably hotter than LMC-SMP 76 ( $T_{\text {eff }} \gtrsim 90$ vs. $\sim 50 \mathrm{kK})$. The absence of low-ionization spectral features supports the idea that these two objects lie in this high temperature regime. Preliminary models computed by us indicate that, in this regime, the high temperatures alone are adequate to reproduce the observed O VI profile, and including reasonable amounts of X-rays in the model atmospheres does not alter this profile significantly. We plan a more detailed investigation of the role of X-rays in the winds of CSPN to be the subject of a future paper.

SMC-SMP 1 (Fig. 13)

Due to uncertainties with the FUSE observations, we have determined the characteristics of this object by mainly relying on wind features in its UV spectrum, using Si IV $\lambda \lambda 1394,1402$, and C IV $\lambda \lambda 1548,51$ as diagnostics. We have also used the far-UV C III $\lambda 1175$ feature for guidance. These features indicate a relatively low terminal wind velocity of $\mathrm{v}_{\infty} \simeq 500 \mathrm{~km} \mathrm{~s}^{-1}$. Because of the limited diagnostics, we have assumed normal, "H-rich" SMC abundances. Models of $T_{\text {eff }} \simeq 37 \mathrm{kK}$ produce acceptable fits to the UV spectrum (cooler temperatures weaken C IV $\lambda \lambda 1548,51$ unacceptably, and hotter temperatures prohibit the Si IV $\lambda \lambda 1394,1402$ and C III $\lambda 1175$ features). However, we are unable to attain acceptable fits of the FUSE spectrum with our stellar models, even when incorporating various hydrogen absorption models. The limited wavelength coverage prevent a determination of $E_{\mathrm{B}-\mathrm{V}}$ from the spectral fitting, so we have simply adopted the value of $E_{\mathrm{B}-\mathrm{V}}=0.16$ (from Meatheringham \& Dopita 1991a, see Table 3). We note that in Fig. 13 what appears to be strong stellar He II emission feature at $\lambda 1640$ is actually multiple narrow emission features (one of which being nebular He II) blended together as a result of the binning for clarity.

SMC-SMP 22 (Fig. 14)

This star has a very high photoionization temperature of $T_{e f f}=200 \mathrm{kK}$ (Table 7). However, we are able to fit the FUSE spectrum well with a SMC-metallicity TLUSTY model of $T_{\text {eff }}=25 \mathrm{kK}(\log g=3.7)$. Most of the detailed structure is from Fe III, which is present in the atmosphere for $T_{\text {eff }} \lesssim 30 \mathrm{kK}$ With $z=0.1 \mathrm{Z}_{\odot}$, the $\mathrm{C}$ III $\lambda 1175$ feature is a bit weak. Using LMC metallicity $\left(z=0.4 \mathrm{Z}_{\odot}\right)$ the line is better fit, but a higher temperature $\left(T_{e f f}=25\right)$ is required to match the Fe III spectrum. We are able to fit its FUSE spectrum without invoking a hot circumstellar molecular gas as found in other MC CSPN.

The photometric SMC catalog of Zaritsky et al. (2002) lists an object 17.4 arcseconds away from our target (coordinates: $05836.77-71366.23$ ) with $\mathrm{B}-\mathrm{V}=-0.26, \mathrm{U}-\mathrm{B}=-0.77$. These colors are consistent with those of an early B-type star, which would have $T_{\text {eff }} \sim 20 \mathrm{kK}$. A main sequence star of this subtype would have $R \simeq 4 \mathrm{R}_{\odot}$ and $\log g \simeq 4$. Using a TLUSTY model with these parameters, and scaling the flux to the distance of the SMC (assuming a foreground reddening of 0.05) results in a far-UV flux level of $\simeq 1 \times 10^{-14} \mathrm{erg} \mathrm{s}^{-1} \mathrm{~cm}^{-2-1}$, about $80 \%$ of the flux levels of our FUSE observations. Scaling its flux by a factor of $\simeq 1.5$ to match the U-magnitude from the catalog $(\mathrm{U}=16.38)$ results in essentially the far-UV flux levels of the FUSE observations. Given that the FUSE flux levels are several times higher than the FOS fluxes, we believe that the most likely explanation is that, due to the significant target drift experienced during the observation of this target $(\$ 2.1)$, the light from the B-type star entered the FUSE aperture to some extent, and the FUSE spectra are dominated by this other object, rather than the target. Thus we exclude this object from subsequent analysis.

\section{DISCUSSION}

In Table 7, we present a combined summary of our findings here with those of HB04. We list temperatures derived from photo-ionization models (Dopita \& Meatheringham 1991a b; Dopita et al. 1997; Vassiliadis et al. 1998b) as well as those from our stellar atmospheres analysis. We also list the measured terminal velocity of the wind, and the morphology of the surrounding PN. In Figs. 15 and 16 we plot our sample along with that of HB04 with the evolutionary tracks of VW94 for LMC and SMC metallicities, respectively (both hydrogen and helium burning tracks are shown). Most of the the LMC sample is seen to lie in the He-burning regime. VW94 found similar results when they plotted their tracks with the CSPN 
samples of Dopita \& Meatheringham (1991a b) (who derived parameters based on photoionization models), and attributed this result to the different timescales of the $\mathrm{H}$ and He-burning tracks. He-burners are expected to take several thousands of years to migrate across the interval $\log L \sim 4.0-2.5 \mathrm{~L}_{\odot}$, whereas the H-burners only take $\lesssim 1000$ years to span the same range. The tracks of VW94 apparently indicate that, as a consequence of their faster evolution to smaller luminosities, the LMC H-burners have already faded below the threshold of FUSE, as our sample represents the expected UV-brightest CSPN.

We found evidence of hot molecular hydrogen in the circumstellar environments of our previous sample of 7 LMC stars (HB04). Here we have made similar findings for our targets. This is further indication that hot $\mathrm{H}_{2}$ in the circumstellar environments of PN is not uncommon (e.g., see Herald \& Bianchi 2002, 2004b for Galactic examples). $\mathrm{H}_{2}$ may exist in clumps, shielded from the intense UV radiation fields by neutral and ionized hydrogen, as appears to be the case in the Helix nebula (Speck et al. 2002). Speck et al. (2002) suggest that these clumps may form after the onset of the PN phase, arising from Rayleigh-Taylor instabilities at either the ionization front or the fast wind shock front. Theoretical models by Aleman \& Gruenwald (2004) show that a significant amount of $\mathrm{H}_{2}$ can survive within the ionized region of $\mathrm{PN}$, and that the ratio of total $\mathrm{H}_{2}$ to hydrogen mass inside the ionized region increases with the temperature of the central star. A correlation between the presence of $\mathrm{H}_{2}$ line emission and the morphology of the PN has been noted by Zuckerman \& Gatley (1988); Kastner et al. (1996) (known as "Gatley's rule").

For the stars analyzed in this paper, our derived effective temperatures are mostly in good agreement with those derived from photoionization models. For SMC-SMP 3, which shows sparse spectral diagnostics, a stellar model with a temperature of about the photoionization temperature $(\sim 95 \mathrm{kK})$ fits the spectra adequately. The temperature derived through spectral modeling (by HPGR) of the enigmatic LMC-SMP 83 is significantly lower than through photoionization models ( $100 \mathrm{kK}$ vs. $170 \mathrm{kK})$.

\section{CONCLUSIONS}

HB04 analyzed 6 LMC CSPN, and here we have analyzed FUSE observations of 4 additional LMC and 2 SMC CSPN. We determined the stellar parameters using the stellar atmosphere code CMFGEN to analyze their FUSE far-UV and HST UV spectra. In some cases, we also modeled the nebular continua (if it contaminates the UV spectra) and the atomic and molecular hydrogen absorption along the sightline (which severely affects the far-UV flux).

To summarize, we find:

- For those objects with photoionization temperatures between $30 \lesssim T \lesssim 100 \mathrm{kK}$, the temperatures determined via stellar modeling are in rough agreement. For LMCSMP 83, our derived effective temperature of $T_{\text {eff }} \simeq$ $95 \mathrm{kK}$, is significantly below the photoionization temperature of $T=170 \mathrm{kK}$. However, this unique object has shown substantial changes in its spectra (and, consequently, its parameters) over the past two decades.

- Most objects often have absorption features in the far-
UV which could be attributed to very hot $(T \gtrsim 2000 \mathrm{~K})$ $\mathrm{H}_{2}$ in their circumstellar environment. These temperatures may be due to the proximity of the nebulae to the star (see theoretical work of Aleman \& Gruenwald 2004), or perhaps due to shocks (as suggested by Speck et al. 2002).

- The majority of our objects have $\mathrm{v}_{\infty} \leq 1000 \mathrm{~km} \mathrm{~s}^{-1}$. Two of the exceptions show signs of chemically enriched winds (LMC-SMP 83 \& LMC-SMP 76). For the other (SMC-SMP 3) the poor data quality prohibit a definitive statement about wind abundances from this spectral analysis.

- We find indications of X-rays in the winds of LMCSMP 76. We are able to attain good fits consistently for the stronger wind features, except for the far-UV O VI $\lambda \lambda 1032,38$ doublet, which is too weak in our models unless X-rays are included in the calculations. Although it has been shown that X-rays are needed to replicate this feature for O and early Bstars (Bianchi \& Garcia 2002; Garcia \& Bianchi 2004; Bianchi et al. 2006), this is the first case of a similar phenomena in CSPN winds.

- These FUSE observations add two cases (LMCSMP 83 and SMC-SMP 22) to the small number of CSPN displaying nebular O VI $\lambda \lambda 1032,38$ (the first case, LMC-SMP 62, was reported by HB04).

By virtue of their membership of the Magellanic Clouds, the uncertainties in their distances is small, which carries over to the derived stellar parameters. This is a great advantage over Galactic CSPN, where the distance is the largest source of uncertainty in the analysis.

Our FUSE observations have allowed us to derive a set of stellar and wind parameters for CSPN of the Magellanic Clouds that are unhampered by the distance uncertainties that plague equivalent Galactic studies. In addition to revealing the flux of the hot star (which is obscured at longer wavelengths by the nebular flux), the far-UV observations also indicate that many of these young CSPN have hot molecular hydrogen in their nebulae, and are thus also giving insight into their circumstellar environments and histories.

We are grateful to the referee, Klaus Werner, for a careful reading of the manuscript and for his constructive comments. We thank John Hillier his help with the CMFGEN code, and Thierry Lanz and Ivan Hubeny for their help with the TLUSTY code. We thank Stephan McCandliss for making his $\mathrm{H}_{2}$ molecular data available. We acknowledge that without the efforts to calculate atomic data of the OPACITY project, this work would not have been possible. The SIMBAD database was used for literature searches. This work has been funded by NASA grants NAG-12294 (FUSE-C056) and NAG-13679 (FUSE-D034). The HST data presented in this paper were obtained from the Multimission Archive at the Space Telescope Science Institute (MAST). STScI is operated by the Association of Universities for Research in Astronomy, Inc., under NASA contract NAS5-26555. 
APPENDIX

\section{APPENDIX: MODEL ATOMS}

For our TLUSTY models, the atomic data used come from TOPBASE, the data-base of the Opacity Project (Cunto et al. 1993). Hydrogen and Helium were treated in NLTE, and the other included species (C, N, O, Ne, Na, Mg, Si, S, Ar, Ca, and Fe) were allowed to contribute to the total number of particles and charge but their opacity contribution was neglected in the model atmosphere calculation.

Ions and the number of levels and superlevels included in the CMFGEN model calculations are listed in able 8 . The atomic data come from a variety of sources, with the Opacity Project (Seaton 1987; Opacity Project Team 1995, 1997), the Iron Project (Pradhan et al. 1996; Hummer et al. 1993), Kurucz (1995) ${ }^{1}$ and the Atomic Spectra Database at NIST Physical Laboratory being the principal sources. Much of the Kurucz atomic data were obtained directly from CfA (Kurucz 1988, 2002). Individual sources of atomic data include the following: Zhang \& Pradhan (1997), Bautista \& Pradhan (1997), Becker \& Butler (1995), Butler et al. (1993), Fuhr et al. (1988), Luo \& Pradhan (1989), Luo et al. (1989), Mendoza (1983, 1995, private communication), Mendoza et al. (1995), Nussbaumer \& Storey (1983, 1984), Peach et al. (1988), Storey (1988, private communication), Tully et al. (1990), and Wiese et al. (1966, 1969). Unpublished data taken from the Opacity Project include: Fe VI data (Butler, K.) Fe VIII data (Saraph and Storey) and C. Mendoza (Fe IX, Fe X).

\section{REFERENCES}

Aleman, I. \& Gruenwald, R. 2004, ApJ, 607, 865

Anderson, L. S. 1989, ApJ, 339, 588

Bautista, M. A. \& Pradhan, A. K. 1997, A\&AS, 126, 365

Becker, S. R. \& Butler, K. 1995, A\&A, 301, 187

Bessell, M. S. 1991, A\&A, 242, 17

Bianchi, L. \& Garcia, M. 2002, ApJ, 581, 610

Bianchi, L., Herald, J., \& Garcia, M. 2006, in The Ultraviolet Universe: Stars from Birth to Death, 26th meeting of the IAU, 16-17 August 2006, Prague, Czech Republic, ed. A. G. de Castro \& M. Barstow, Vol. 4, JD04, \#36

Bianchi, L., Vassiliadis, E., \& Dopita, M. 1997, ApJ, 480, 290

Bohlin, R. C., Savage, B. D., \& Drake, J. F. 1978, ApJ, 224, 132

Bouret, J.-C., Lanz, T., Hillier, D. J., Heap, S. R., Hubeny, I., Lennon, D. J., Smith, L. J., \& Evans, C. J. 2003, ApJ, 595, 1182

Butler, K., Mendoza, C., \& Zeippen, C. J. 1993, Phys. Rev. B, 26, 4409

Cardelli, J. A., Clayton, G. C., \& Mathis, J. S. 1989, ApJ, 345, 245

Chu, Y.-H., Gruendl, R. A., \& Guerrero, M. A. 2004, in Asymmetric Planetary nebulae III; ASP Conference Series, Vol. 313, ed. M. Meixner, J. Kastner, B. Balick, \& N. Soker (San Fransisco: ASP), 259

Cohen, D. H., de Messières, G. E., MacFarlane, J. J., Miller, N. A., Cassinelli, J. P., Owocki, S. P., \& Liedahl, D. A. 2003, ApJ, 586, 495

Cunto, W., Mendoza, C., Ochsenbein, F., \& Zeippen, C. J. 1993, A\&A, 275, 5

Dopita, M. A., Ford, H. C., Lawrence, C. J., \& Webster, L. 1985, ApJ, 296, 390

Dopita, M. A. \& Meatheringham, S. J. 1991a, ApJ, 367, 115

-. 1991b, ApJ, 377, 480

Dopita, M. A., Meatheringham, S. J., Webster, B. L., \& Ford, H. C. 1988, ApJ, 327, 639

Dopita, M. A., Vassiliadis, E., Meatheringham, S. J., Bohlin, R. C., Ford, H. C., Harrington, J. P., Wood, P. R., Stecher, T. P., \& Maran, S. P. 1996 , ApJ, 460, 320

Dopita, M. A., Vassiliadis, E., Wood, P. R., Meatheringham, S. J., Harrington, J. P., Bohlin, R. C., Ford, H. C., Stecher, T. P., \& Maran, S. P. 1997, ApJ, 474, 188

Dufour, R. J. 1984, in IAU Symp., Vol. 108, Structure and Evolution of the Magellanic Clouds, ed. S. van den Bergh \& K. S. de Boer (Dordrecht: Kluwer), 353

Feast, M. W. 1991, in IAU Symp., Vol. 148, The Magellanic Clouds, ed. R. Haynes \& D. Milne (Dordrecht: Kluwer), 1

Fitzpatrick, E. L. 1999, PASP, 111, 63

Fuhr, J. R., Martin, G. A., \& Wiese, W. L. 1988, in J. Phys. Chem. Ref. Data, Vol. Supp. 4 (New York: Am. Chem. Soc. \& AIP), 17

Garcia, M. \& Bianchi, L. 2004, ApJ, 606, 497

Grevesse, N. \& Sauval, A. J. 1998, Space Sci. Rev., 85, 161

Guerrero, M. A., Chu, Y.-H., Gruendl, R. A., Williams, R. M., \& Kaler, J. B. 2001, ApJ, 553, L55

Hamann, W.-R. \& Koesterke, L. 1998, A\&A, 333, 251

Hamann, W.-R., Koesterke, L., \& Wessolowski, U. 1993, A\&A, 274, 397

Hamann, W.-R., Peña, M., Gräfener, M., \& Ruiz, M. T. 2003, A\&A, 409, 969

Harries, T. J., Hilditch, R. W., \& Howarth, I. D. 2003, MNRAS, 339, 157

Henry, R. B. C., Liebert, J., \& Boroson, T. A. 1989, ApJ, 339, 872

Herald, J. E. \& Bianchi, L. 2002, ApJ, 580, 434

-. 2004a, ApJ, 611, 294

-. 2004b, ApJ, 609, 378

\footnotetext{
${ }^{1}$ See http://cfa-www.harvard.edu/amdata/ampdata/amdata.shtml
}

Hillier, D. J., Lanz, T., Heap, S. R., Hubeny, I., Smith, L. J., Evans, C. J., Lennon, D. J., \& Bouret, J. C. 2003, ApJ, 588, 1039

Hillier, D. J. \& Miller, D. L. 1998, ApJ, 496, 407

-. 1999, ApJ, 519, 354

Hubeny, I. \& Lanz, T. 1995, ApJ, 439, 875

Hughes, M. P., Thompson, A. R., \& Colvin, R. S. 1971, ApJS, 23, 323

Hummer, D. G., Berrington, K. A., Eissner, W., et al. 1993, A\&A, 279, 298

Kastner, J. H., Weintraub, D. A., Gatley, I., Merrill, K. M., \& Probst, R. G. 1996, ApJ, 462, 777

Kurucz, R. L. 1988, in Transactions of the International Astronomical Union, ed. D. R. Schultz, P. S. Krstic, \& F. Ownby, Vol. XXB (Dordrecht: Kluwer), 168

Kurucz, R. L. 1995, CD ROM 23, Atomic Line Data (Cambridge: Smithsonian Astrophysical Obs.)

Kurucz, R. L. 2002, in AIP Conf. Proc., Vol. 183, Atomic and Molecular Data and Their Applications, ed. D. R. Schultz, P. S. Krstic, \& F. Ownby (Melville: AIP), 636

Luo, D. \& Pradhan, A. K. 1989, Phys. Rev. B, 22, 3377

Luo, D., Pradhan, A. K., Saraph, H. E., Storey, P. J., \& Yan, Y. 1989, Phys. Rev. B, 22, 389

Marigo, P., Girardi, L., Groenewegen, M. A. T., \& Weiss, A. 2001, A\&A, 378,958

Martins, F., Schaerer, D., Hillier, D. J., Meynadier, F., Heydari-Malayeri, M., \& Walborn, N. R. 2005, A\&A, 441, 735

Meatheringham, S. J. \& Dopita, M. A. 1991a, ApJS, 75, 407

-. 1991b, ApJS, 76, 1085

Meatheringham, S. J., Dopita, M. A., \& Morgan, D. H. 1988, ApJ, 329, 166

Mendoza, C., Eissner, W., Le Dourneuf, M., \& Zeippen, C. J. 1995, Phys. Rev. B, 28, 3485

Monk, D. J., Barlow, M. J., \& Clegg, R. E. S. 1988, MNRAS, 234, 583

Moos, H. W., Cash, W. C., Cowie, L. L., et al. 2000, ApJ, 538, 1

Nussbaumer, H. \& Storey, P. J. 1983, A\&A, 126, 75

-. 1984, A\&AS, 56, 293

Opacity Project Team. 1995, The Opacity Project, Vol. 1 (Bristol: Institute of Physics Publications)

-. 1997, The Opacity Project, Vol. 2 (Bristol: Institute of Physics Publications)

Owocki, S. P., Castor, J. I., \& Rybicki, G. B. 1988, ApJ, 335, 914

Owocki, S. P., Cranmer, S. R., \& Blondin, J. M. 1994, ApJ, 424, 887

Peña, M., Hamann, W.-R., Ruiz, M. T., Peimbert, A., \& Peimbert, M. 2004, A\&A, 419, 583

Peach, G., Saraph, H. E., \& Seaton, M. J. 1988, Phys. Rev. B, 21, 3669

Pradhan, A. K., Zhang, H. L., Nahar, S. N., Romano, P., \& Baustista, M. A. 1996, BAAS, 189, 7211

Raymond, J. C. \& Smith, B. W. 1977, ApJS, 35, 419

Sahnow, D. J., Moos, M. W., Ake, T. B., et al. 2000, ApJ, 538, 7

Schmutz, W., Hamann, W.-R., \& Wessolowski, U. 1989, A\&A, 210, 236

Schulz, N. S., Canizares, C., Huenemoerder, D., \& Tibbets, K. 2003, ApJ, 595,365

Seaton, M. J. 1987, Phys. Rev. B, 20, 6363

Shaw, R. A., Stanghellini, S., \& Mutchler, M. 2001, ApJ, 548, 727

Speck, A. K., Meixner, M., Fong, D., McCullough, P. R., Moser, D. E., \& Ueta, T. 2002, ApJ, 123, 346

Stanghellini, L., Blades, J. C., Osmer, S. J., Barlow, M. J., \& Liu, X.-W. 1999, ApJ, 510, 687 
Tully, J. A., Seaton, M. J., \& Berrington, K. A. 1990, Phys. Rev. B, 23, 3811 Vassiliadis, E., Dopita, M. A., Bohlin, R. C., Harrington, J. P., Ford, H. C., Meatheringham, S. J., Wood, P. R., Stecher, T. P., \& Maran, S. P. 1996, ApJS, 105, 375

-. 1998a, ApJS, 503, 253

Vassiliadis, E., Dopita, M. A., Meatheringham, S. J., Bohlin, R. C., Ford, H. C., Harrington, J. P., Wood, P. R., Stecher, T. P., \& Maran, S. P. 1998b, ApJ, 503, 253

Vassiliadis, E. \& Wood, P. R. 1994, ApJ, 92, 125

Wiese, L. L., Smith, M. W., \& Glennon, B. M. 1966, NSRDS-NBS 4 , Vol. 1, Atomic Transition Probabilites (Washington, D.C.: US Government Printing Office)
Wiese, L. L., Smith, M. W., \& Miles, B. M. 1969, NSRDS-NBS 22, Vol. 2, Atomic Transition Probabilites (Washington, D.C.: US Government Printing Office)

Zaritsky, D., Harris, J., Thompson, I. B., Grebel, E. K., \& Massey, P. 2002, AJ, 123,855

Zhang, H. L. \& Pradhan, A. K. 1997, A\&AS, 126, 373

Zuckerman, B. \& Gatley, I. 1988, ApJ, 324, 501 


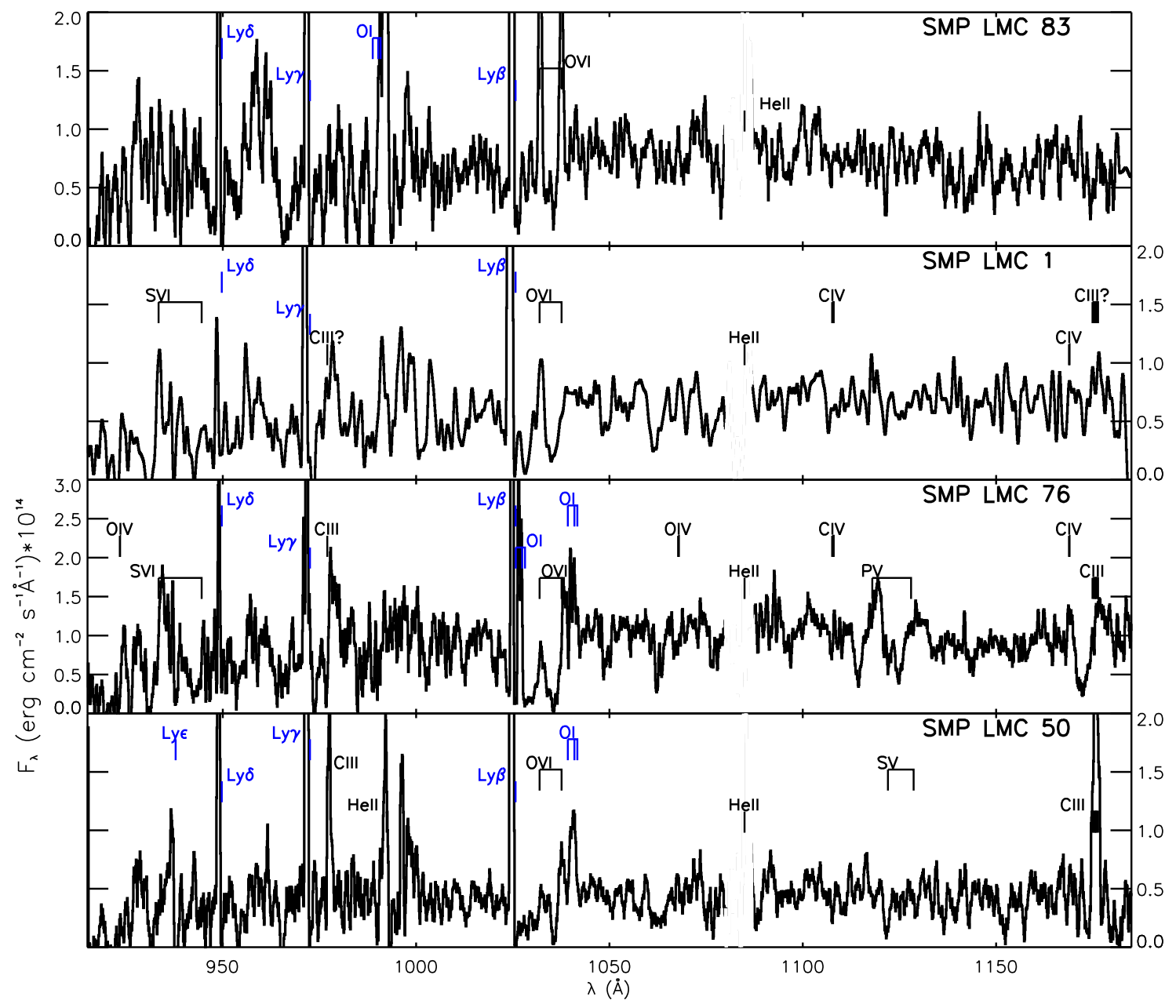

FIG. 1.- FUSE spectra of our LMC sample, velocity shifted to the rest frame of the central star (Table 3, and re-binned to a resolution of $0.10 \AA$. . The more prominent stellar features and nebular emission lines are marked by black labels, airglow features are marked by the blue labels. Most objects seem to display wind lines to some extent. Nebular O VI emission appears in the spectra of LMC-SMP 83. 


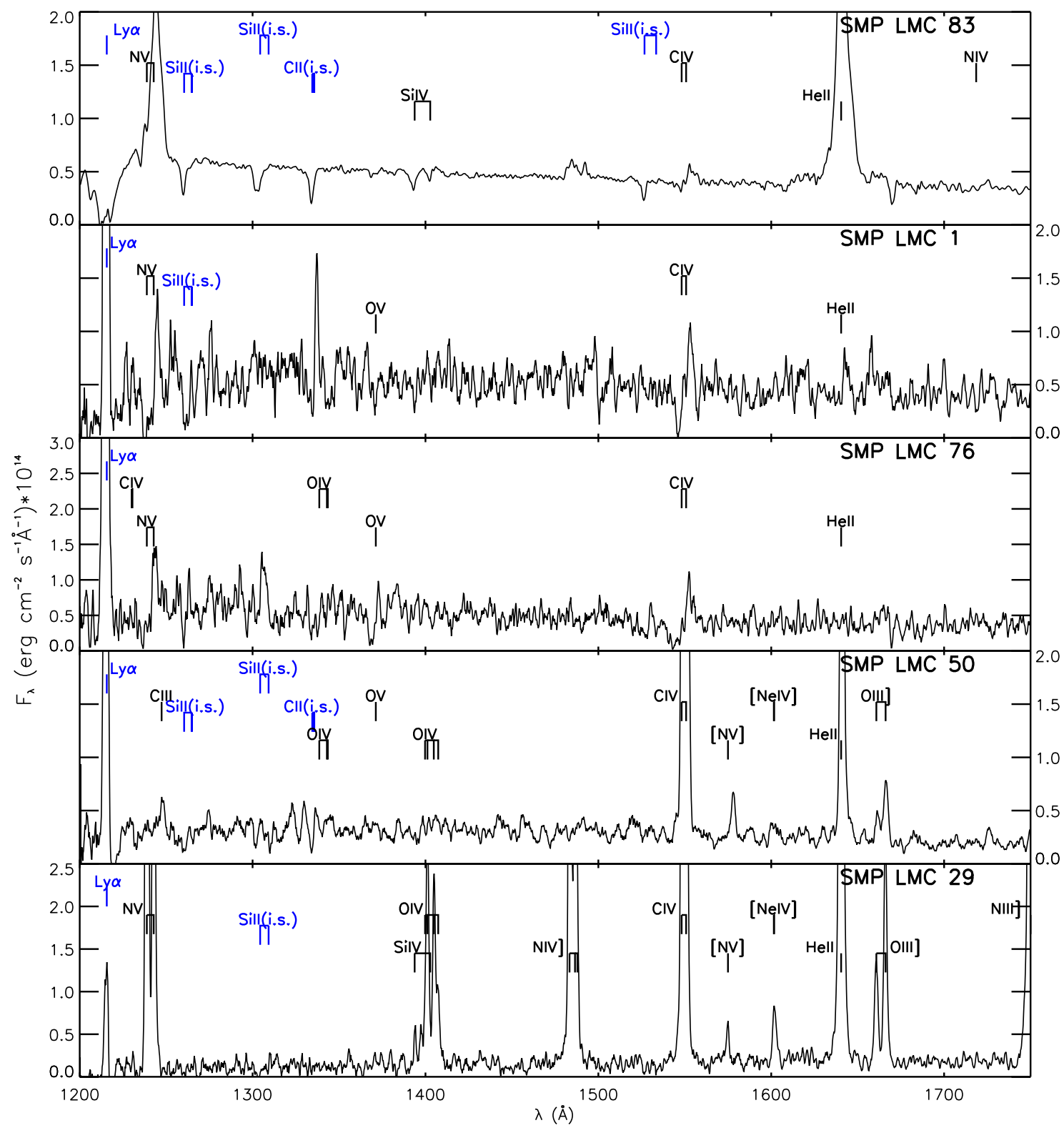

FIG. 2.- FOS and STIS (for LMC-SMP 83) spectra of our LMC sample, velocity shifted to the rest frame of the central star (Table 3 , and re-binned to a resolution of $0.25 \AA$. The more prominent stellar features and nebular emission lines are marked with black labels, interstellar absorption features are marked with blue/gray labels. Most objects display wind lines to some extent. C IV $\lambda \lambda 1548-51$ appears as a P-Cygni profile in most. The spectra of LMC-SMP 50 and LMC-SMP 29 show emission lines from nebulae in high states of ionization. 


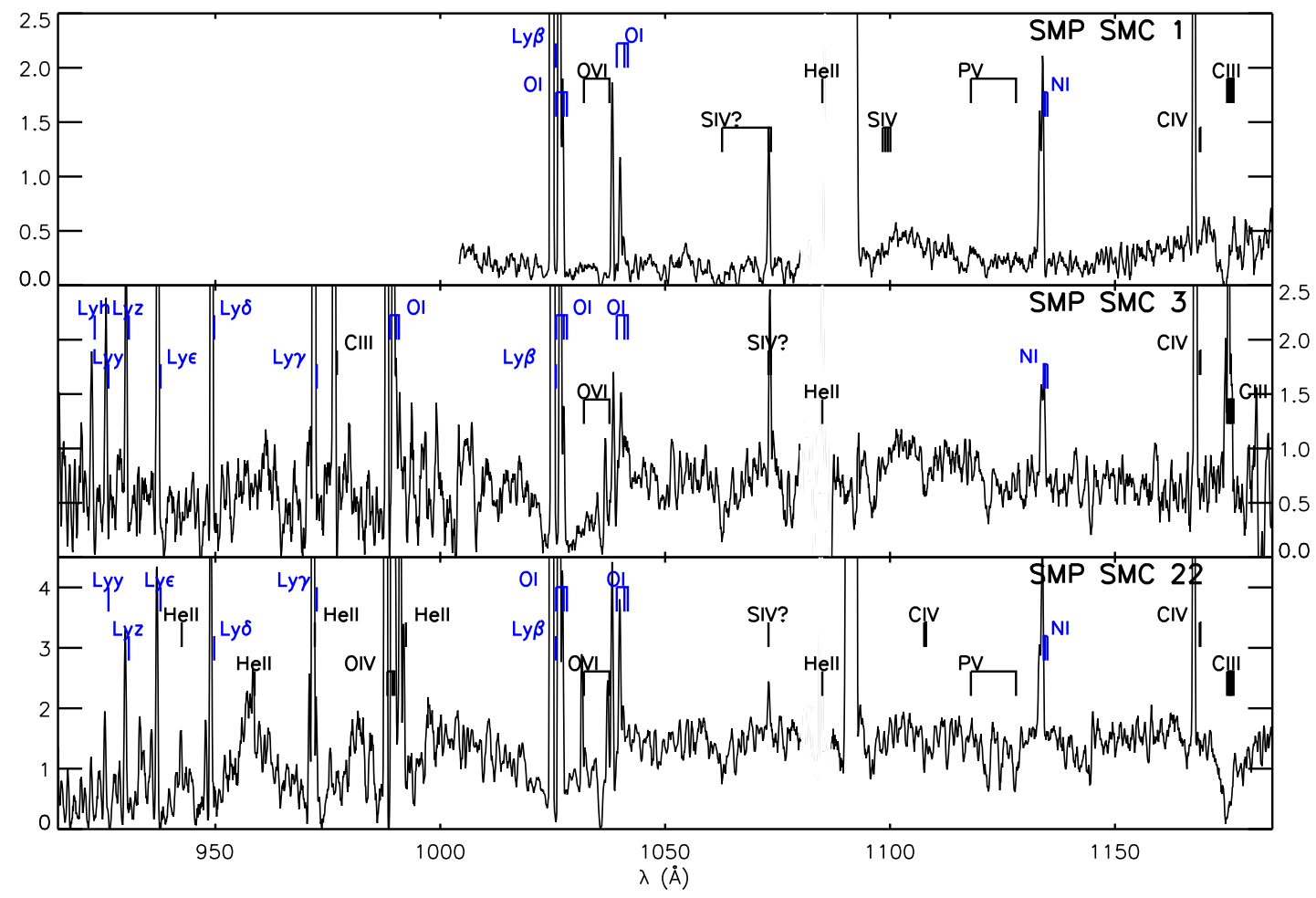

FIG. 3. - FUSE spectra of our SMC sample, velocity shifted to the rest frame of the central star (Table 3), and re-binned to a resolution of $0.10 \AA$. . The more prominent stellar features and nebular emission lines are marked by black labels, airglow features are marked by the blue/gray labels. Note the O VI $\lambda \lambda 1032-38$ nebular emission features of SMC-SMP 22. 


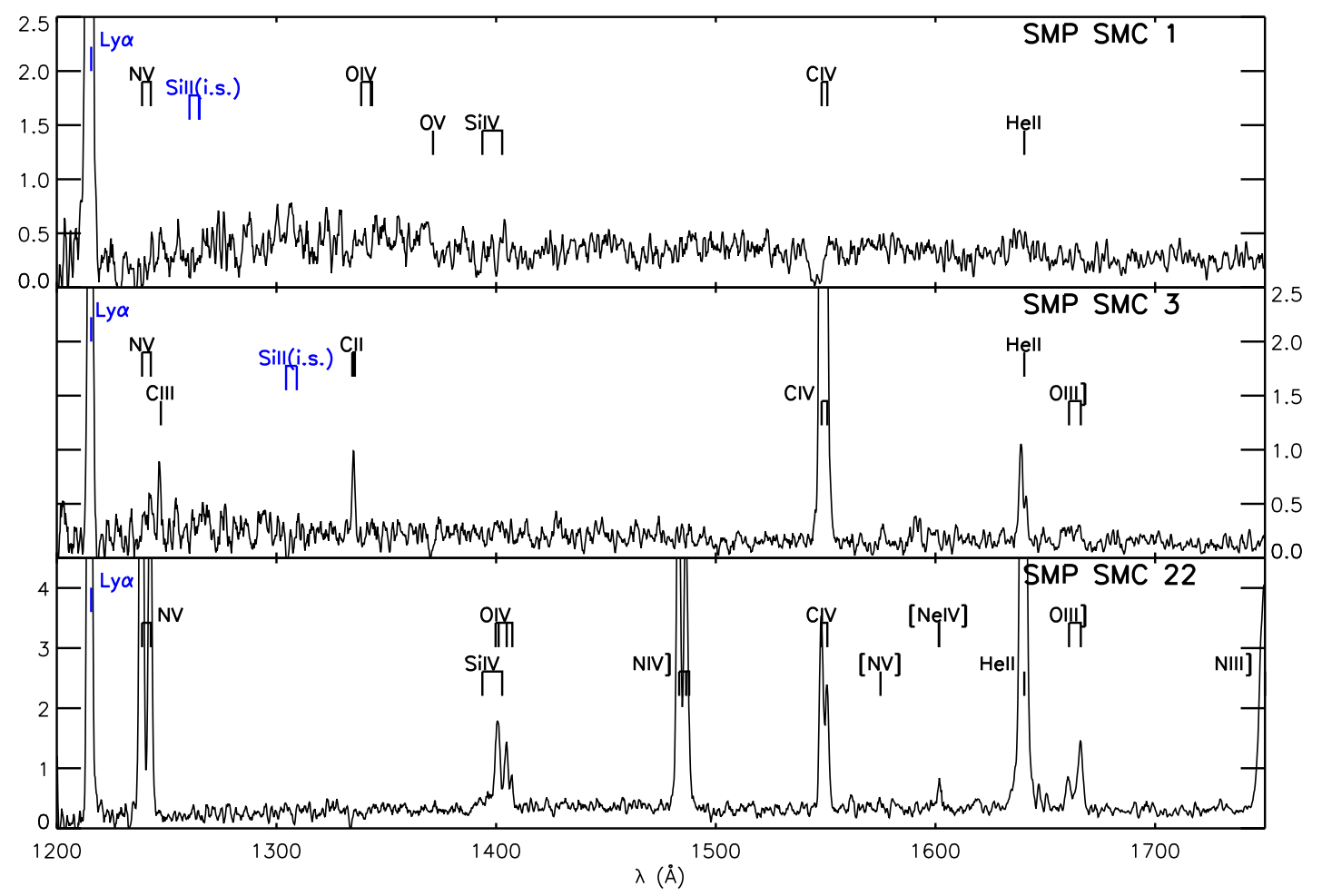

FIG. 4. - UV spectra (FOS) of our SMC sample, velocity shifted to the rest frame of the central star (Table 3), and re-binned to a resolution of 0.25 $\mathrm{A}$. The more prominent stellar features and nebular emission lines are marked with black labels, airglow lines or interstellar absorption features are marked with blue/gray labels. 


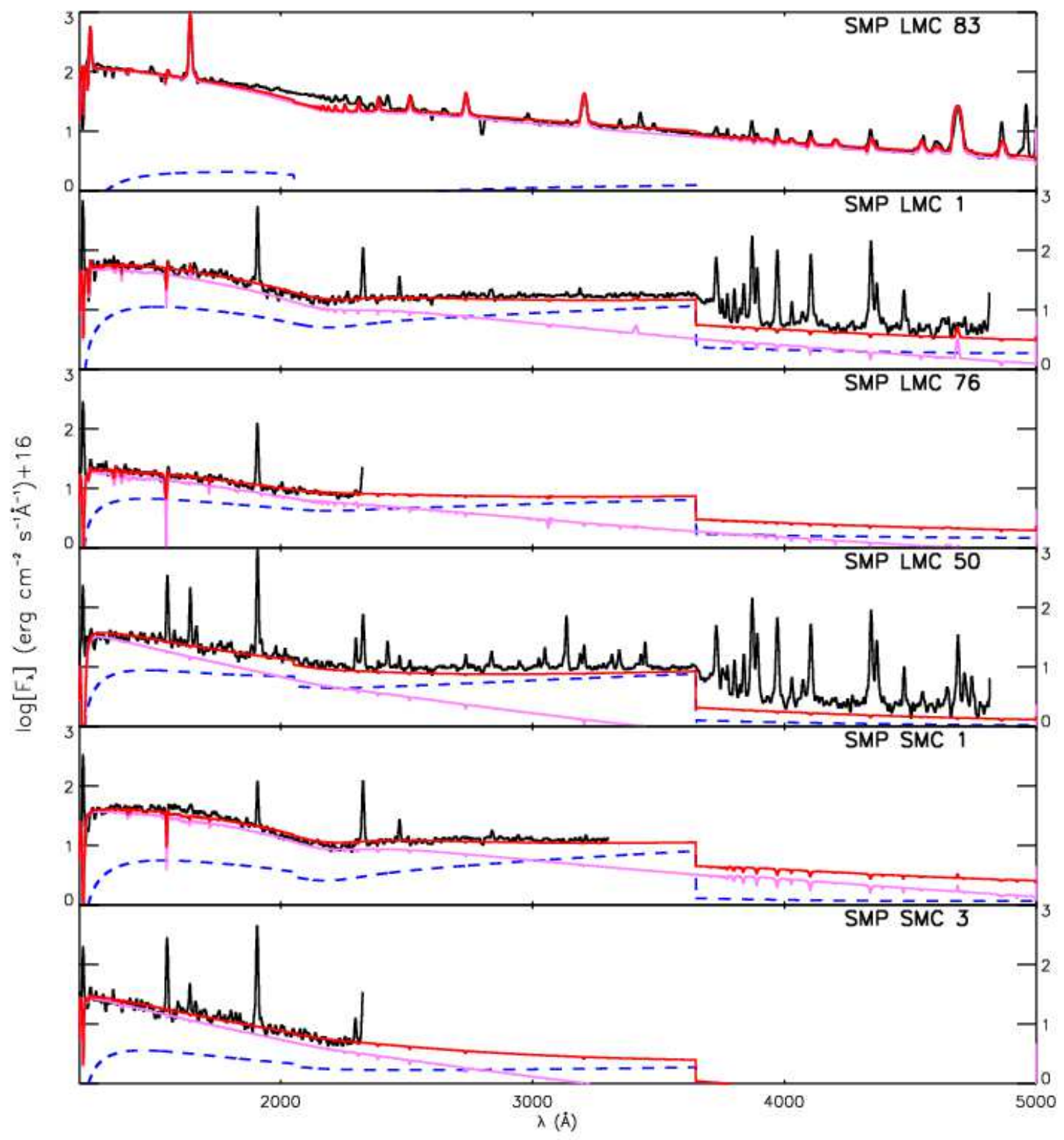

FIG. 5.- The MC CSPN spectra (black) are shown along with our stellar (pink/grey) and nebular continuum models (blue dashed). The sum of the models is shown by the red line. All models have been reddened with our determined values for $E_{\mathrm{B}-\mathrm{V}}$ (Table 6), and the effects of hydrogen absorption (Table 5 , have been applied. The spectra have been convolved with a $5 \AA$ Aaussian for clarity. Note the logarithmic flux scale. 


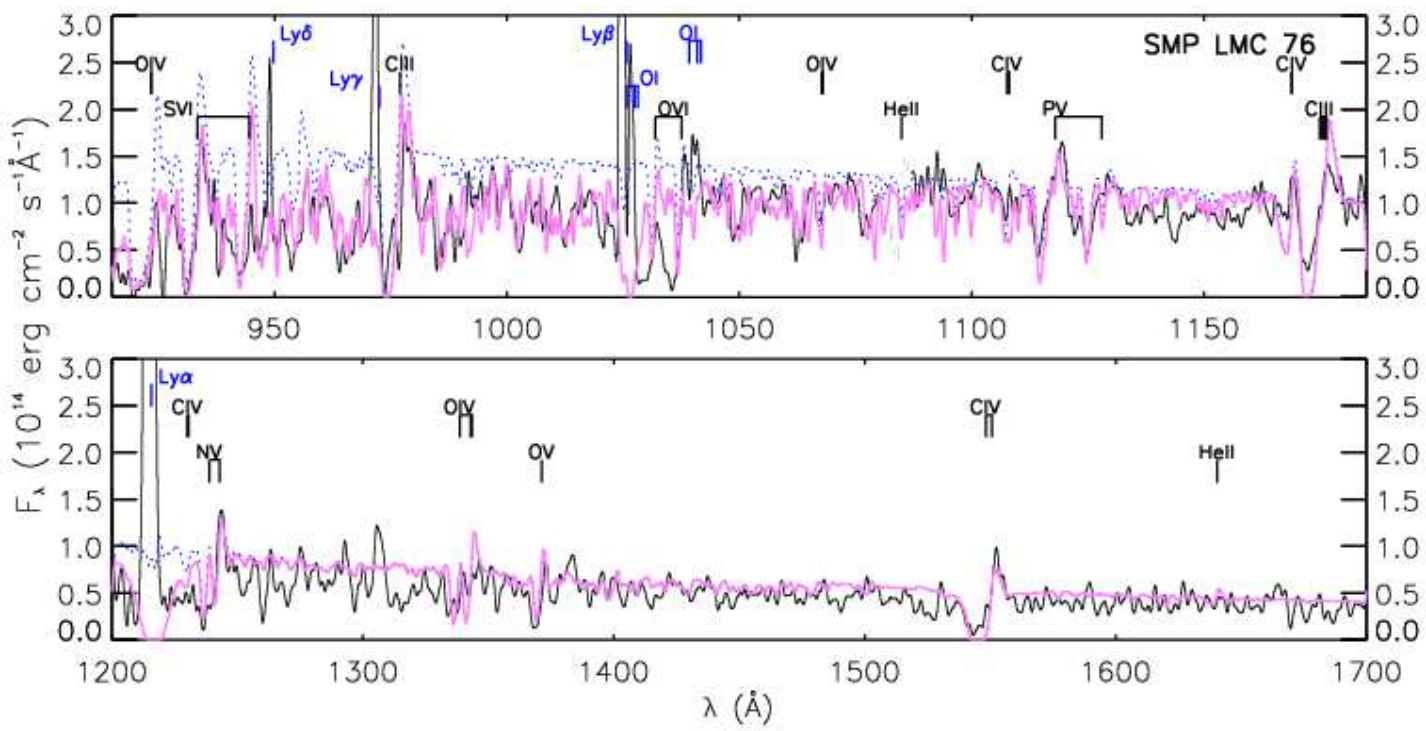

FIG. 6. - LMC-SMP 76: The observations are shown (black) along with our stellar model, with and without our hydrogen absorption models applied (pink/gray and blue dotted, respectively). The FUSE spectra have been convolved with a 0.6 A Gaussian for clarity.

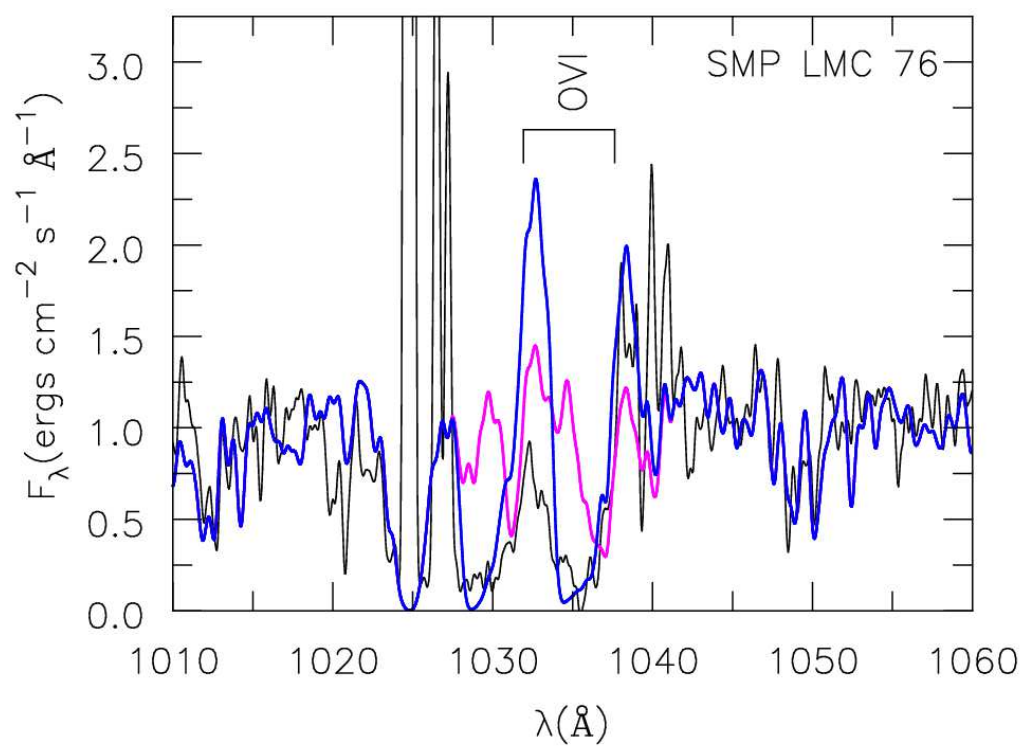

FIG. 7.- LMC-SMP 76: The observations are shown (black) along with two models, one without X-rays (pink/gray) and one with X-rays of strength $\log \mathrm{L}_{\mathrm{x}} / \mathrm{L}_{\text {bol }} \lesssim-7.0$ (blue/dark grey). Without X-rays, the model fails to duplicate the strong P-Cygni absorption trough caused by O VI $\lambda \lambda 1032,38$. Including $\mathrm{X}$-rays in the model calculations strengthens the $\mathrm{O}$ VI feature while not affecting other diagnostics. 


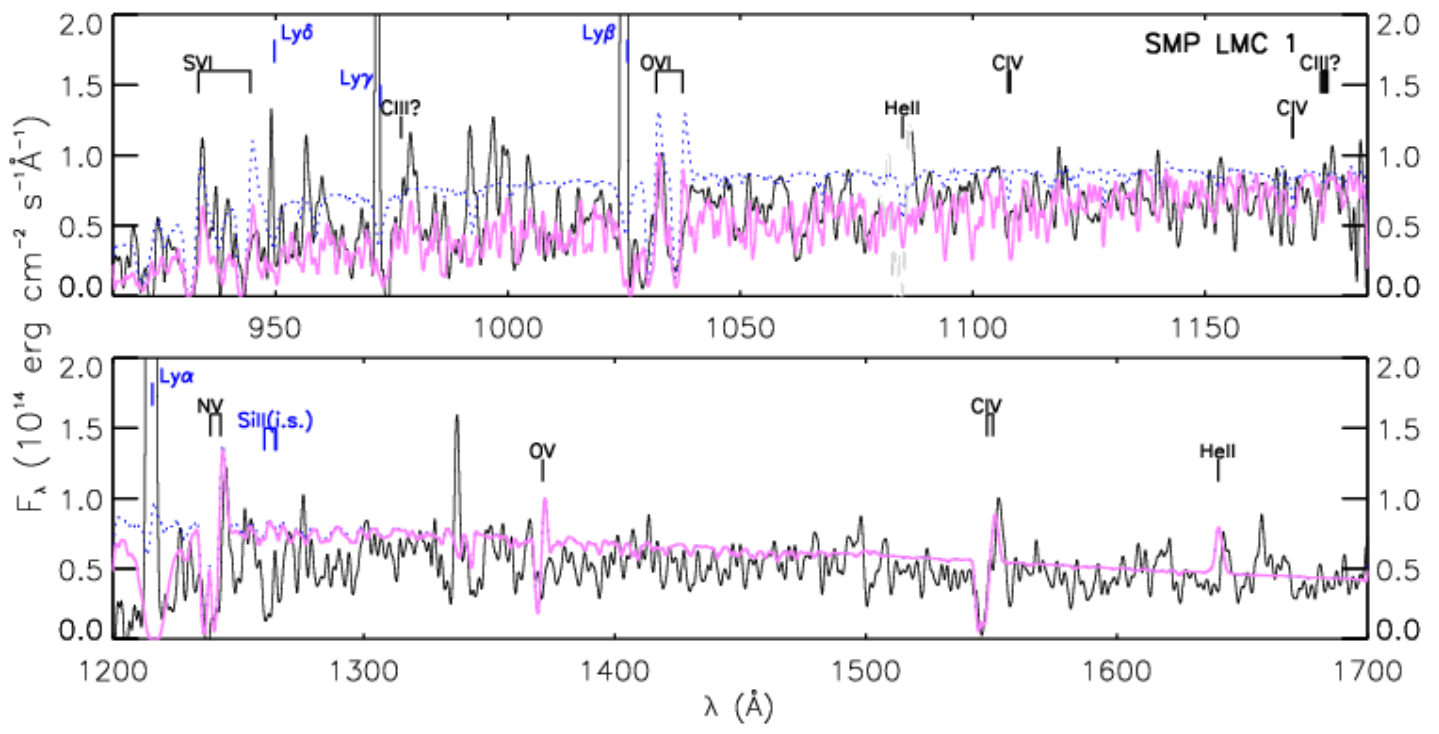

FIG. 8.- LMC-SMP 1: Description follows that of Fig. 6

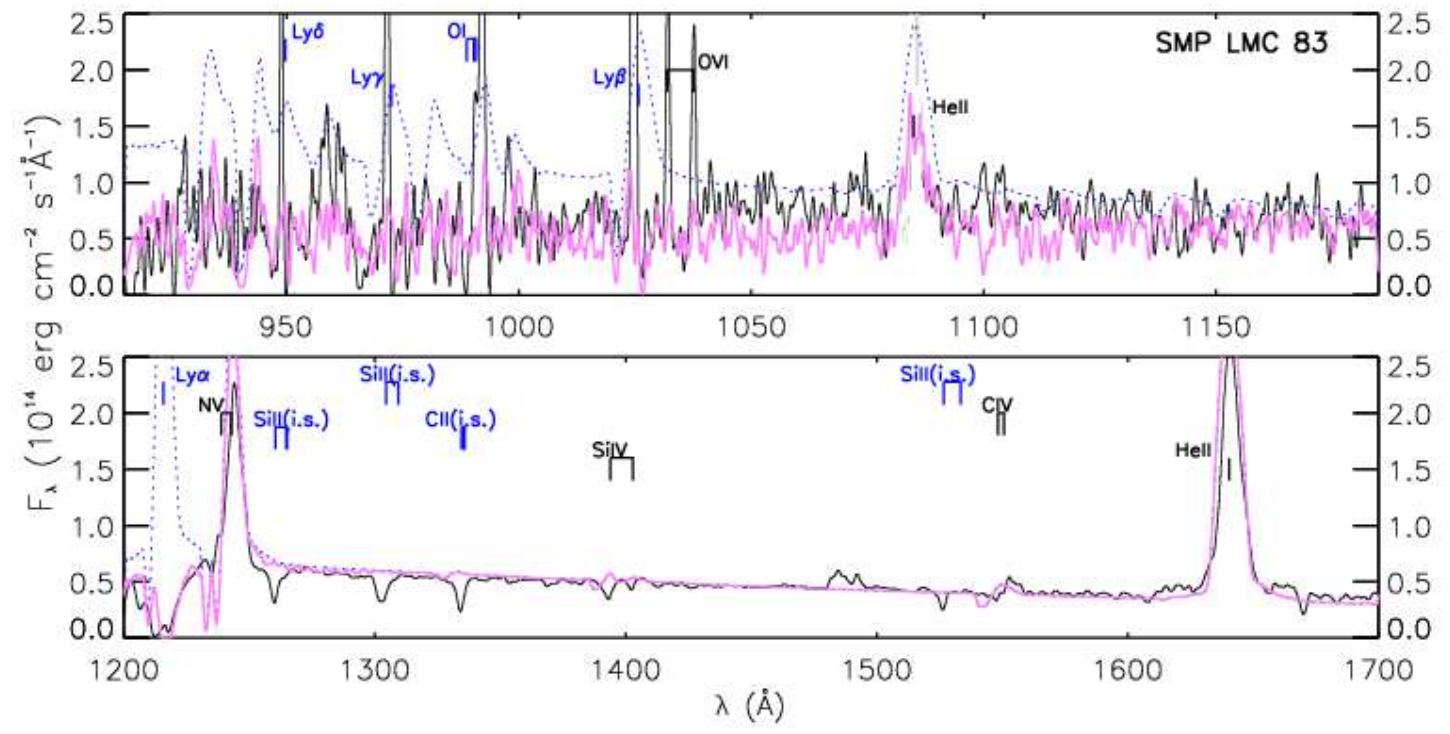

FIG. 9. - LMC-SMP 83: Description follows that of Fig. 6 


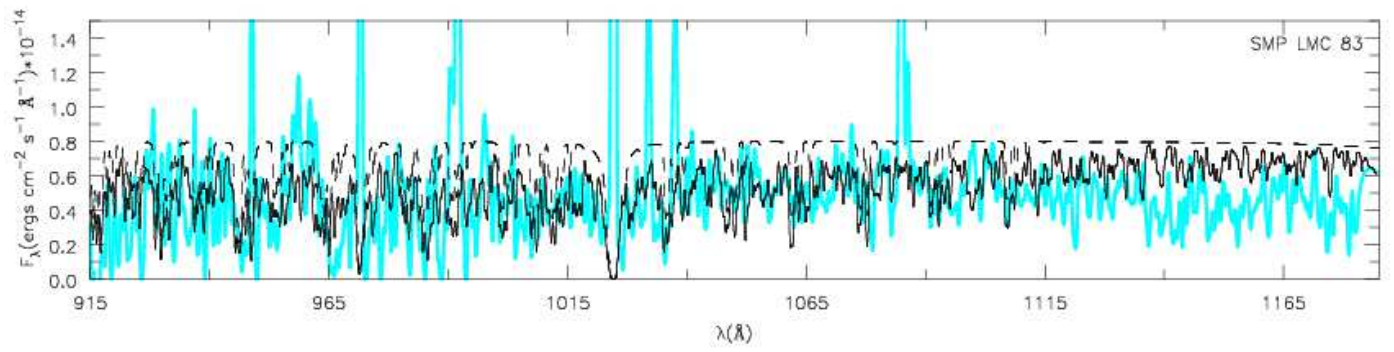

FIG. 10.- LMC-SMP 83: A portion of the FUSE spectrum is shown (black) along with a flat model continuum, both with only an interstellar hydrogen absorption component applied (dashed) and with an additional hot circumstellar $\mathrm{H}_{2}$ component applied (green).

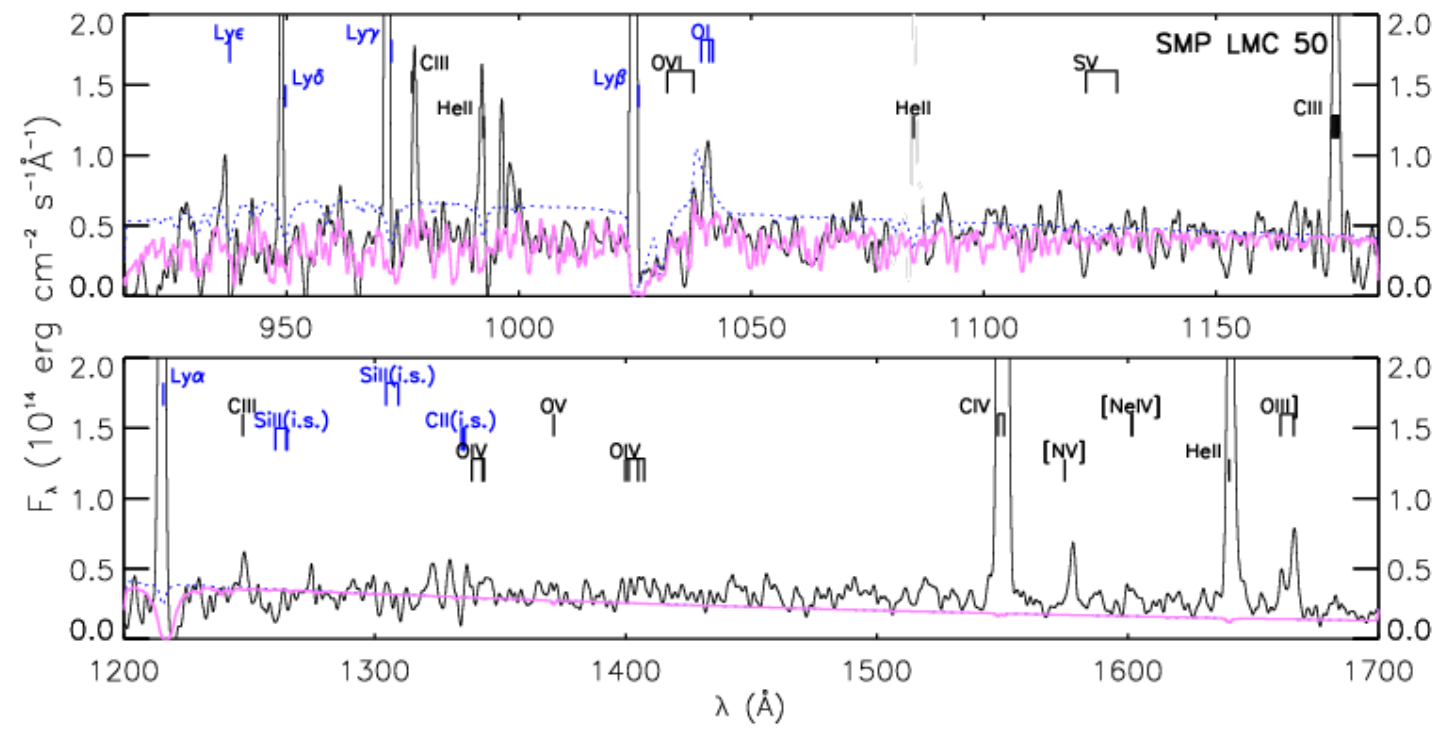

FIG. 11.- LMC-SMP 50: Description follows that of Fig.6 


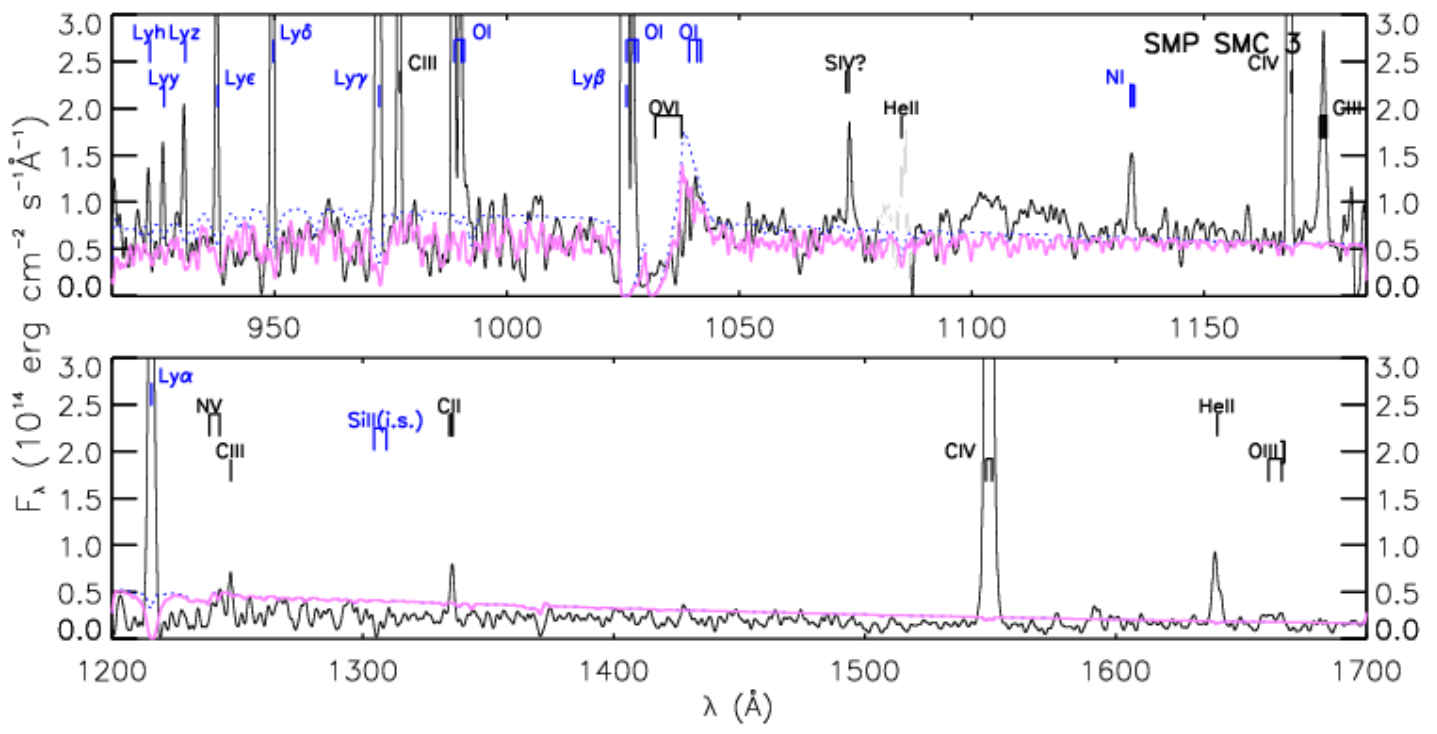

FIG. 12.- SMC-SMP 22: Description follows that of Fig. 6

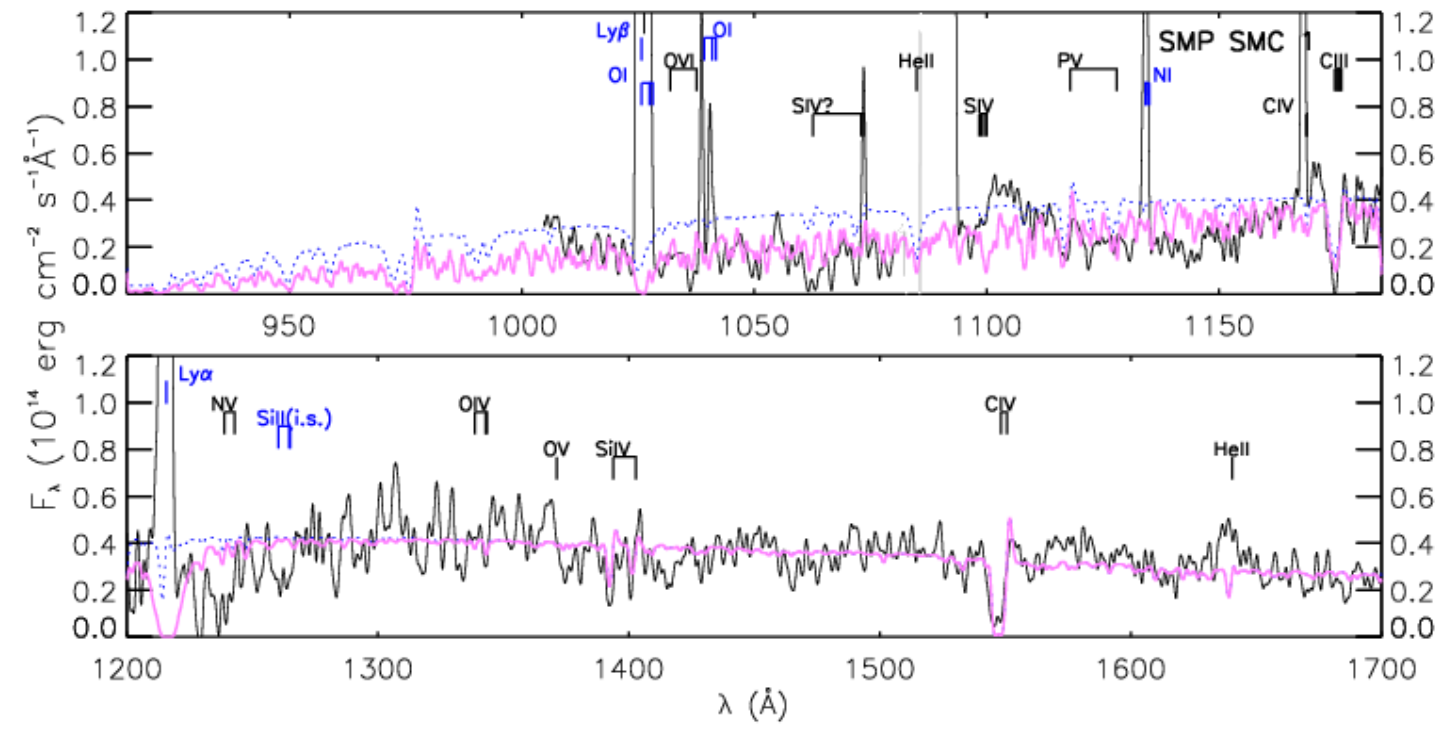

FIG. 13.- SMC-SMP 1: Description follows that of Fig. 6 


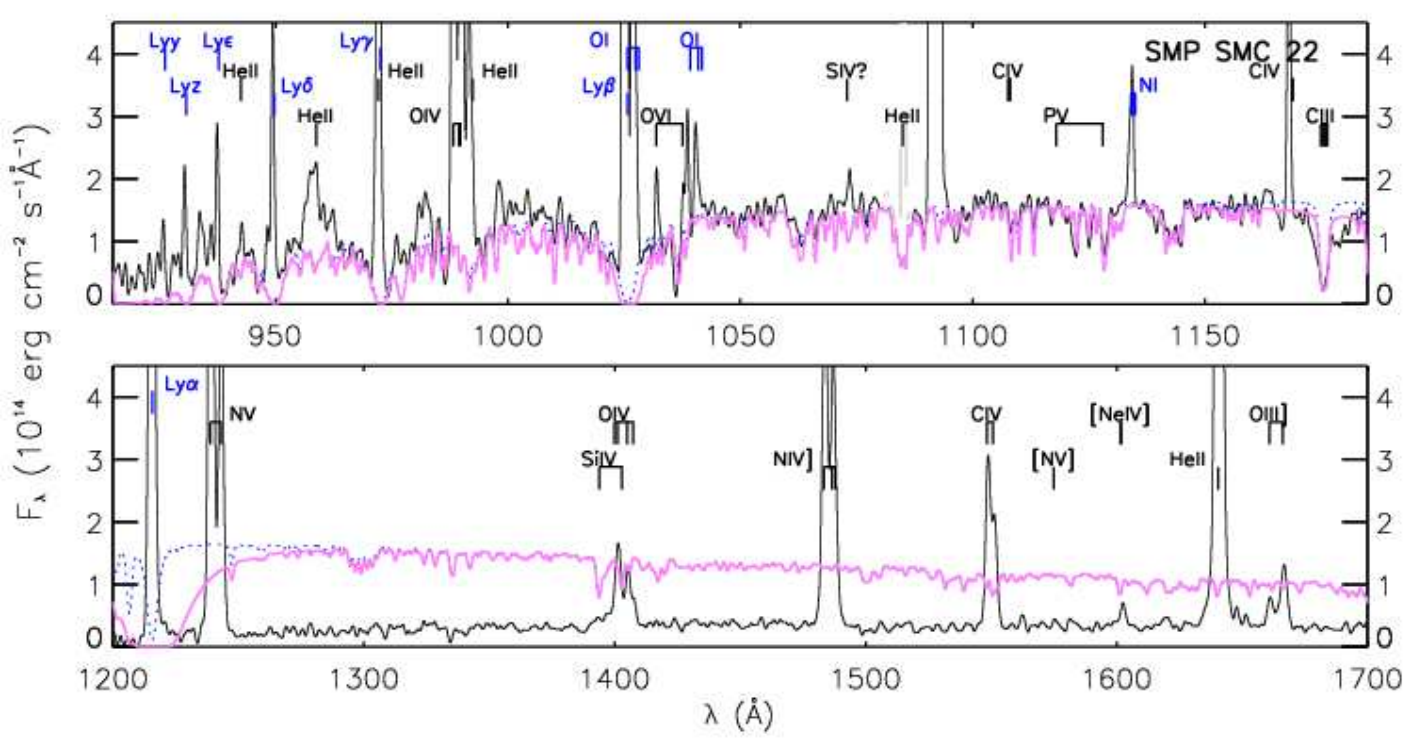

FIG. 14.- SMC-SMP 22: Description follows that of Fig. 6 Note: this observation was apparently contaminated by a B-star in the aperture, and so does not represent the true spectrum of SMC-SMP 22 (this is the reason for the flux discrepancy between the far-UV and UV spectra). 


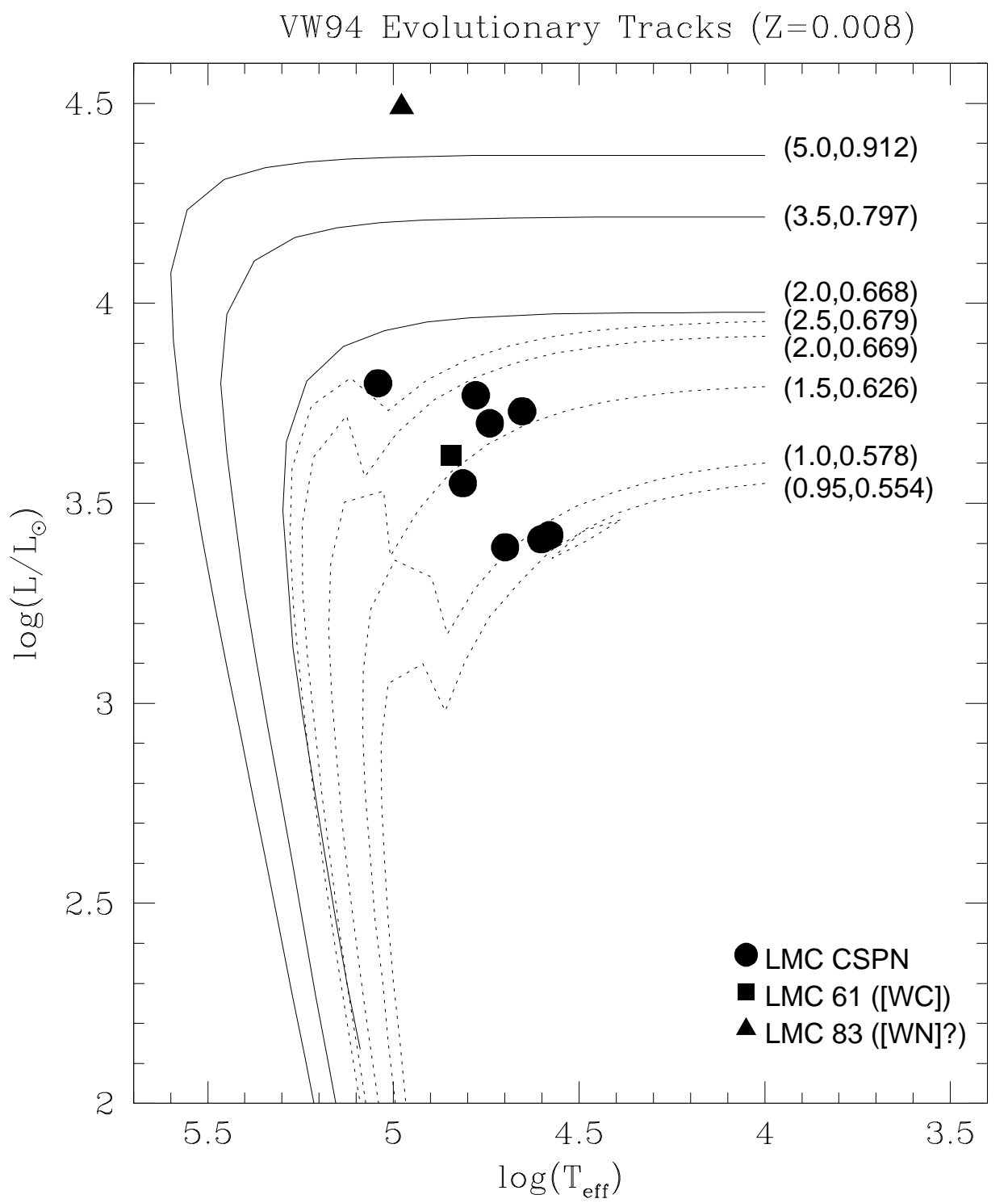

FIG. 15. - HR diagram of our LMC sample and that of HB04. Evolutionary tracks $(\mathrm{Z}=0.008)$ of VW94 are shown, labeled with their initial and final masses, with the $\mathrm{H}$ and He-burning tracks denoted by the solid and dotted lines, respectively. Note our adopted model metallicity corresponds to $\mathrm{Z}=0.006$. 


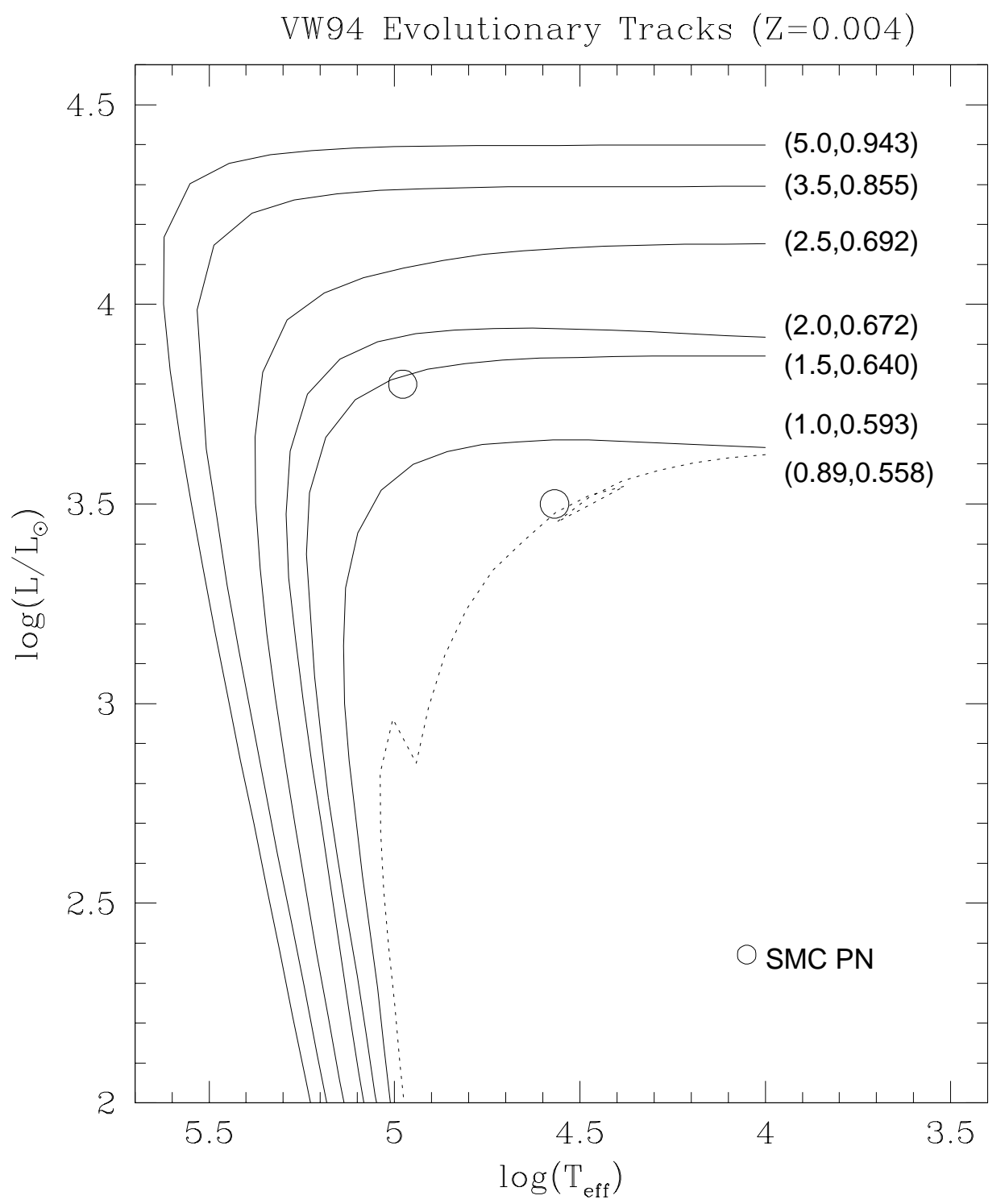

FIG. 16. - HR diagram of our SMC sample. Evolutionary tracks $(\mathrm{Z}=0.004)$ of VW94 are shown, labeled with their initial and final masses, with the H and He-burning tracks denoted by the solid and dotted lines, respectively. Note our adopted model metallicity corresponds to $\mathrm{Z}=0.0016$. 
TABLE 1

FUSE C056 AND D034 PROGRAM STARS, AND FUSE DETECTOR SEGMENTS UTILIZED

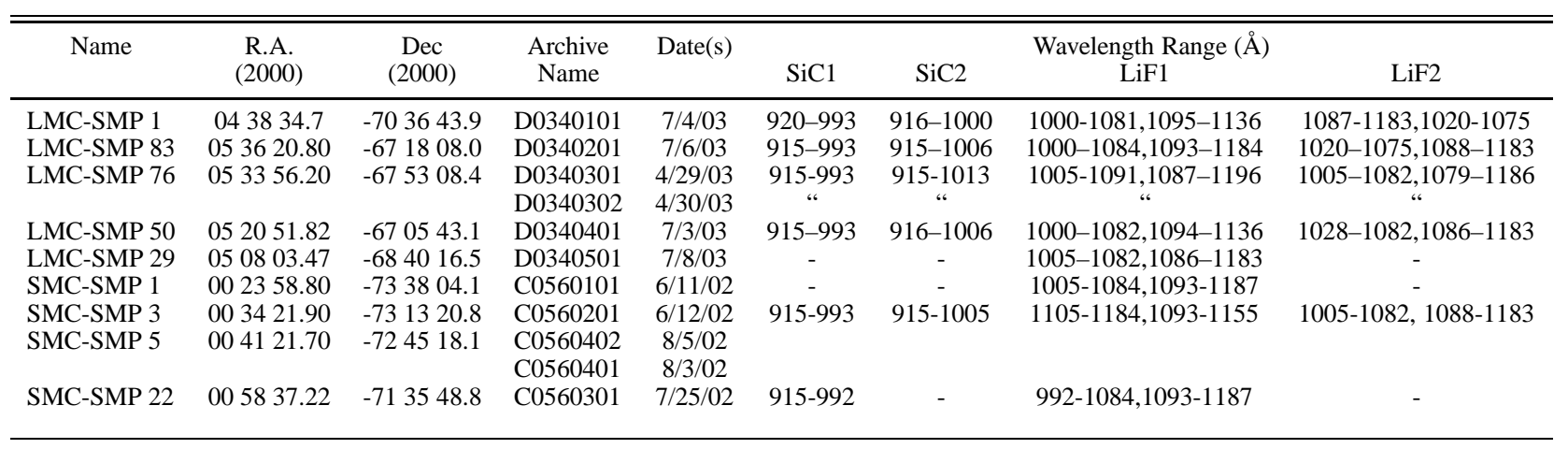

TABLE 2

ARCHIVE UV DATA SETS (FOS AND STIS)

\begin{tabular}{cccccc}
\hline \hline Name & Instrument & Dataset & Date & Grating & $\lambda(\AA)$ \\
\hline LMC-SMP 76 & FOS & Y1C10B03T & $9 / 22 / 93$ & G130H & $1182-1600$ \\
& FOS & Y1C10B03T & $9 / 22 / 93$ & G190H & $1600-2330$ \\
LMC-SMP 83 & STIS & O5IM01010 & $02 / 04 / 00$ & G140L & $1182-1720$ \\
& STIS & O5IM01020 & $02 / 04 / 00$ & G230L & $1720-3150$ \\
& STIS & O5IM02010 & $02 / 04 / 00$ & G430L & $3150-5200$ \\
& STIS & O5IM02010 & $02 / 04 / 00$ & G430L & $3150-5200$ \\
& STIS & O55R01010 & $01 / 08 / 99$ & G140L & $1182-1720$ \\
& STIS & O55R01020 & $01 / 08 / 99$ & G230L & $1720-3150$ \\
& STIS & O55R01030 & $01 / 08 / 99$ & G430L & $3150-5200$ \\
& STIS & O55R02020 & $01 / 08 / 99$ & G750L & $5265-10260$ \\
LMC-SMP 1 & FOS & Y3EV0106P & $11 / 15 / 96$ & G130H & $1182-1600$ \\
& FOS & Y3EV0103P & $11 / 15 / 96$ & G190H & $1600-2300$ \\
& FOS & Y3EV0104P & $11 / 15 / 96$ & G270H & $2300-3280$ \\
& FOS & Y3EV0105P & $11 / 15 / 96$ & G400H & $3280-4822$ \\
LMC-SMP 50 & FOS & Y3EV0805T & $11 / 10 / 96$ & G130H & $1182-1600$ \\
& FOS & Y3EV0802T & $11 / 10 / 96$ & G190H & $1600-2300$ \\
& FOS & Y3EV0803T & $11 / 10 / 96$ & G270H & $2300-3280$ \\
& FOS & Y3EV0804T & $11 / 10 / 96$ & G400H & $3280-4822$ \\
LMC-SMP 29 & FOS & Y2Y00204T & $10 / 22 / 95$ & G130H & $1182-1600$ \\
& FOS & Y2Y00205T & $10 / 22 / 95$ & G190H & $1600-2300$ \\
& FOS & Y2Y00206T & $10 / 22 / 95$ & G270H & $2300-3280$ \\
& FOS & Y2Y00207T & $10 / 22 / 95$ & G400H & $3280-4822$ \\
\hline SMC-SMP 1 & FOS & Y1C10103T & $5 / 3 / 93$ & G130H & $1182-1600$ \\
& FOS & Y1C10104T & $5 / 3 / 93$ & G190H & $1600-2330$ \\
SMC-SMP 3 & FOS & Y1C10203T & $11 / 26 / 93$ & G130H & $1182-1600$ \\
& FOS & Y1C10204T & $11 / 26 / 93$ & G190H & $1600-2330$ \\
SMC-SMP 5 & FOS & Y2Y00904T & $6 / 12 / 96$ & G130H & $1182-1600$ \\
& FOS & Y2Y00905T & $6 / 12 / 96$ & G190H & $1600-2330$ \\
& FOS & Y2Y00906T & $6 / 12 / 96$ & G270H & $2300-3280$ \\
SMC-SMP 22 & FOS & Y2Y00907T & $6 / 12 / 96$ & G400H & $3280-4822$ \\
& FOS & Y2N30104T & $3 / 20 / 95$ & G130H & $1182-1600$ \\
& FOS & Y2N30105T & $3 / 20 / 95$ & G190H & $1600-2330$ \\
\hline
\end{tabular}


TABLE 3

NebUlar PARAMETERS

\begin{tabular}{|c|c|c|c|c|c|c|c|c|c|c|c|}
\hline Name & $\begin{array}{l}\theta^{a a} \\
\left({ }^{\prime \prime}\right)\end{array}$ & $\begin{array}{l}r_{n e b}^{b b} \\
(\mathrm{pc})\end{array}$ & $\begin{array}{c}v_{\exp }(\mathrm{OIII} / \mathrm{OII})^{\mathrm{a}} \\
\quad\left(\mathrm{km} \mathrm{s}^{-1}\right)\end{array}$ & $\begin{array}{l}\tau_{d y n}^{c c} \\
(\mathrm{kyr})\end{array}$ & $\begin{array}{c}n_{e}([\mathrm{OII} / \mathrm{SII}])^{\mathrm{b}} \\
\quad\left(10^{3} \mathrm{~cm}^{-3}\right)\end{array}$ & $\begin{array}{c}T_{e}\left(\mathrm{O}^{+2} / \mathrm{N}^{+1}\right)^{b} \\
(\mathrm{kK})\end{array}$ & $\begin{array}{c}\log \left(F_{H \beta}\right)^{h} \\
()\end{array}$ & $\begin{array}{l}E_{\mathrm{B}-\mathrm{V}}\left(\mathrm{c}_{H \beta}\right)^{b} \\
\quad(\mathrm{mag})\end{array}$ & $\mathrm{He} / \mathrm{H}^{c}$ & $\mathrm{He}^{2+} / \mathrm{He}^{+}$ & $\begin{array}{c}v_{L S R} \\
\left(\mathrm{~km} \mathrm{~s}^{-1}\right)\end{array}$ \\
\hline LMC-SMP 1 & $0.33^{s}$ & 0.04 & $17.2 / 23.1$ & 2.3 & $3.44 / 3.32$ & $11.5 /-$ & $-12.46(-\mathbf{1 2 . 5})$ & 0.11 & 0.146 & $-e(\mathbf{0 . 0})$ & $209.1^{a}$ \\
\hline LMC-SMP 29 & 0.68 & $0.083^{d}$ & $35.9 / 49.3$ & 2.3 & $5.46 / 4.06$ & $20.0 /-$ & -12.71 & 0.05 & 0.160 & $1.53^{e}$ & $228.2^{a}$ \\
\hline LMC-SMP 50 & $0.64^{s}$ & 0.08 & $35.0 / 55.7$ & 2.2 & $5.37 /-$ & $11.2 /-$ & $-12.71(\mathbf{- 1 2 . 8 1 )}$ & 0.04 & 0.120 & $0.23^{e}$ & $284.1^{a}$ \\
\hline LMC-SMP 76 & $<0.16$ & $<0.02$ & $29.0^{x x} / 32.9$ & 0.7 & $13.7 /-$ & $12.0 /-$ & -12.54 & 0.22 & 0.075 & $-^{e}(\mathbf{0 . 0})$ & $262.8^{a}$ \\
\hline LMC-SMP 83 & 2.34 & $0.292^{d, x}$ & $82.9^{x x} / 80.9$ & 3.4 & $2.46 / 2.22$ & $17.4 / 11.7$ & -12.65 & 0.10 & 0.137 & $1.15^{e}$ & $274.5 / 300^{k}$ \\
\hline SMC-SMP 1 & 0.31 & $0.045^{d}$ & $15.4 / 16.1$ & 2.9 & $9.59 /-$ & $10.7 /-$ & $-12.77(-12.89)$ & 0.16 & 0.080 & ${ }^{-e} / 0.155^{g}$ & $138.0^{j}$ \\
\hline SMC-SMP 3 & 0.49 & $0.072^{d}$ & $32.9 /-$ & 2.1 & $1.96 /-$ & $13.5 /-$ & $-13.06(-\mathbf{1 2 . 7 8})$ & 0.03 & 0.160 & $0.07^{e} / 1.66^{g}$ & $98.2^{j}$ \\
\hline SMC-SMP 5 & 0.42 & $0.061^{d}$ & $29.2 / 43.6$ & 2.0 & $3.7 / 3.34$ & $14.5 /-$ & -12.81 & 0.07 & 0.130 & $0.60^{e}$ & $101.2^{j}$ \\
\hline SMC-SMP 22 & 0.52 & $0.075^{d}$ & $50.9 / 51.2$ & 1.5 & $5.51 / 2.33$ & $26.6 / 11.6$ & -12.88 & 0.11 & $0.135^{g}$ & & $144.2^{j}$ \\
\hline $\begin{array}{l}\text { REFERENCES. } \\
\text { Dopita et al. }[1996) \\
\text { aa Calculated from } \\
\text { bb From their "D( }(\mathrm{ec} \\
\text { bc } \tau_{d y n}=r_{\text {neb }} / v_{\text {exp }} \\
\text { dd } \text { Line fit with two } \\
\text { ee } \text { From [S II] [Me: } \\
\text { xx Multiple compon } \\
\text { xxx Avg. of } 3 \text { comp }\end{array}$ & 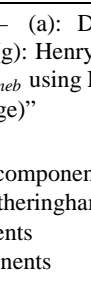 & $\begin{array}{l}\text { ita et al. } \\
\text { t al. }(1989 \\
\text { IC/SMC }\end{array}$ & $\begin{array}{l}\text { 88) (b): Meatheri } \\
\text { h): } \text { Meatheringham } \\
\text { ances of } 50.6 / 60.0\end{array}$ & $\begin{array}{l}\operatorname{ham} 8 \\
\text { al. } 1 \\
\text { c }\end{array}$ & opita (j): 1 (1991a b) & $\begin{array}{l}\text { Dopita \& Mec } \\
\text { 885) (k): Peña }\end{array}$ & $\begin{array}{l}\text { ringham 1991a b } \\
\text { 1. (2004) (s): Shaw }\end{array}$ & $\begin{array}{l}\text { 1): Vassiliadi } \\
\text { al. } 2001)(\mathrm{x})\end{array}$ & $\begin{array}{l}\text { al. (19 } \\
\text { prox o }\end{array}$ & $\begin{array}{l}\text { (e): Monk e } \\
\text { ee vals given }\end{array}$ & 1. (1988) (f): \\
\hline
\end{tabular}

TABLE 4

ABUNDANCES OF GRID MODELS

\begin{tabular}{ccccccccccc}
\hline \hline Grid & $X_{H}$ & $X_{H e}$ & $X_{C}$ & $X_{N}$ & $X_{O}$ & $X_{S}$ & i & $X_{P}$ & $X_{S}$ & $X_{F e}$ \\
\hline LMC H-deficient & - & 0.54 & 0.37 & 0.01 & 0.08 & $2.80 \times 10^{-4}$ & $2.45 \times 10^{-6}$ & $1.53 \times 10^{-4}$ & $5.44 \times 10^{-4}$ \\
LMC H-rich & 0.71 & 0.28 & $1.22 \times 10^{-3}$ & $4.40 \times 10^{-4}$ & $3.82 \times 10^{-3}$ & $2.80 \times 10^{-4}$ & $2.45 \times 10^{-6}$ & $1.53 \times 10^{-4}$ & $5.44 \times 10^{-4}$ \\
SMC H-rich & 0.71 & 0.28 & $3.05 \times 10^{-4}$ & $1.10 \times 10^{-4}$ & $9.55 \times 10^{-4}$ & $7.00 \times 10^{-5}$ & $6.12 \times 10^{-7}$ & $3.65 \times 10^{-5}$ & $1.36 \times 10^{-4}$ \\
\hline
\end{tabular}

TABLE 5

$\mathrm{H}$ I AND $\mathrm{H}_{2}$ PARAMETERS

\begin{tabular}{|c|c|c|c|c|c|c|c|}
\hline \multirow[b]{2}{*}{ Star } & \multicolumn{2}{|c|}{$\mathrm{H} \mathrm{I}^{I S+c i r c}$} & \multicolumn{2}{|l|}{$\mathrm{H}_{2}{ }^{I S}$} & \multicolumn{2}{|c|}{$\mathrm{H}_{2}{ }^{\text {circ }}$} & \multirow[b]{2}{*}{$\begin{array}{c}E_{\mathrm{B}-\mathrm{V}}(\mathrm{H} \mathrm{I}) \\
(\mathrm{mag})\end{array}$} \\
\hline & $\begin{array}{l}\log N \\
\left(\mathrm{~cm}^{-2}\right)\end{array}$ & $\begin{array}{c}T \\
(\mathrm{~K})\end{array}$ & $\begin{array}{l}\log N \\
\left(\mathrm{~cm}^{-2}\right)\end{array}$ & $\begin{array}{c}T \\
(\mathrm{~K})\end{array}$ & $\begin{array}{l}\log N \\
\left(\mathrm{~cm}^{-2}\right)\end{array}$ & $\begin{array}{c}T \\
(\mathrm{~K})\end{array}$ & \\
\hline LMC-SMP 76 & $21.00_{-0.3}^{+0.3}$ & 80 & $18.70_{-0.3}^{+0.3}$ & 80 & $16.70_{-0.0}^{+0.0}$ & $2000 \pm 1000$ & 0.2 \\
\hline LMC-SMP 1 & $20.70_{-0.0}^{+0.0}$ & 80 & $18.00 \pm 1.0$ & 80 & $17.00_{-0.4}^{+0.4}$ & $3000 \pm 1500$ & 0.1 \\
\hline LMC-SMP 50 & $20.40_{-0.4}^{+0.3}$ & 80 & $19.7_{-0.3}^{+0.3}$ & 80 & $16.70_{-0.7}^{+0.3}$ & $3000 \pm 1500$ & 0.05 \\
\hline LMC-SMP 83 & $20.80_{-0.4}^{+0.4}$ & 80 & $16.70_{-0.7}^{+0.3}$ & 80 & $16.70_{-0.4}^{+0.7}$ & $2000 \pm 1000$ & 0.04 \\
\hline LMC-SMP 29 & -0.4 & - & -0.1 & - & -0.4 & - & - \\
\hline SMC-SMP 1 & 21.9 & 80 & - & - & - & - & - \\
\hline SMC-SMP 3 & 21.7 & 80 & $19.40_{-0.4}^{+0.3} 2.1$ & 80 & $16.70_{-0.4}^{+0.3}$ & $2000 \pm 1000$ & 1.0 \\
\hline SMC-SMP 22 & $20.00_{-0.3}^{+0.3}$ & 80 & $16.70_{-0.4}^{+0.3}$ & 80 & $--^{-0.4}$ & - & 0.02 \\
\hline
\end{tabular}

TABLE 6

Derived Stellar Parameters

\begin{tabular}{|c|c|c|c|c|c|c|c|c|}
\hline Name & $\begin{array}{c}\text { Model } \\
\text { Abundance }\end{array}$ & $\begin{array}{l}T_{e f f} \\
(\mathrm{kK})\end{array}$ & $\begin{array}{l}\log L \\
\left(\mathrm{~L}_{\odot}\right)\end{array}$ & $\begin{array}{c}R_{*} \\
\left(\mathrm{R}_{\odot}\right)\end{array}$ & $\begin{array}{c}\log \dot{M} \\
\left(\mathrm{M}_{\odot} \mathrm{yr}^{-1}\right)\end{array}$ & $\begin{array}{c}\mathrm{v}_{\infty} \\
\left(\mathrm{km} \mathrm{s}^{-1}\right)\end{array}$ & $\begin{array}{l}\log g \\
(\mathrm{cgs})\end{array}$ & $\begin{array}{r}E_{\mathrm{B}-\mathrm{V}} \\
(\mathrm{mag})\end{array}$ \\
\hline LMC-SMP 83 & LMC 83 & 95 & $4.49_{-0.21}^{+0.17}$ & $0.47 \pm 0.1$ & $-5.71_{-0.16}^{+0.13}$ & 1600 & 6.0 & 0.08 \\
\hline LMC-SMP 1 & H-poor & $65_{-10}^{+5}$ & $3.61_{-0.20}^{+0.08}$ & $0.70_{-0.05}^{+0.10}$ & $\begin{array}{l}-7.52_{-0.21}^{+0.20} \\
-10\end{array}$ & $800 \pm 100$ & 5.0 & 0.13 \\
\hline LMC-SMP 1 & H-rich & $65_{-10}^{+5}$ & $3.46_{-0.20}^{+0.08}$ & $0.66_{-0.04}^{+0.10}$ & $-7.46_{-0.21}^{+0.21}$ & $800 \pm 100$ & 5.0 & 0.13 \\
\hline LMC-SMP 76 & H-poor & $50 \pm 5$ & $3.39_{-0.22}^{+0.29}$ & $0.67 \pm 0.1$ & $-7.49_{-0.33}^{+0.31}$ & $1250 \pm 250$ & 4.7 & 0.03 \\
\hline LMC-SMP 50 & H-rich & $110 \pm 10$ & $3.80^{+0.26}$ & $0.22^{+0.03}$ & $-8.2^{-0.30}$ & $500 \pm 100$ & 6.0 & 0.04 \\
\hline LMC-SMP 29 & - & - & -0.34 & -0.04 & -0.20 & - & - & 0.05 \\
\hline SMC-SMP 1 & H-rich & $37_{-2}^{+3}$ & $3.5_{-0.2}^{+0.3}$ & $1.3 \pm 0.2$ & $-7.31 \pm 0.10$ & $500 \pm 100$ & 4.9 & 0.16 \\
\hline SMC-SMP 3 & H-rich & $95 \pm 5$ & $3.8_{-0.2}^{+0.3}$ & $0.32 \pm 0.05$ & $-7.8 \pm 0.1$ & $\geq 2000$ & 5.7 & 0.03 \\
\hline
\end{tabular}


TABLE 7

SUMMARY TABLE

\begin{tabular}{|c|c|c|c|c|}
\hline Name & $\begin{array}{l}T_{e f f}^{a a} \\
(\mathrm{kK})\end{array}$ & $\begin{array}{l}T_{p h o t}^{b b} \\
(\mathrm{kK})\end{array}$ & $\begin{array}{c}\mathrm{v}_{\infty}{ }^{a a} \\
\left(\mathrm{~km} \mathrm{~s}^{-1}\right)\end{array}$ & Morphology \\
\hline LMC-SMP 2 & 38 & 39 & 700 & Spherical $^{c}$, Round $^{a}$ \\
\hline LMC-SMP 85 & 40 & 45 & 700 & Round $^{a}$ \\
\hline LMC-SMP 62 & 45 & 127 & 1000 & Bipolar w/central condensation ${ }^{b}$ \\
\hline LMC-SMP 76 & 50 & 50 & 1250 & Spherical ${ }^{c}$, Round $^{a}$ \\
\hline LMC-SMP 67 & 55 & 46 & 1000 & Bipolar w/central condensation $^{b}$ \\
\hline LMC-SMP 23 & 60 & 65 & 1100 & $\operatorname{Ring}^{b}$, Round $^{a}$ \\
\hline LMC-SMP 1 & 65 & 66 & 800 & \\
\hline LMC-SMP 61 & 70 & 59 & 1300 & Spherical $^{b}$ \\
\hline LMC-SMP 50 & 110 & 100 & 500 & Elliptical (bipolar core?) ${ }^{d}$ \\
\hline LMC-SMP 83 & 95 & 170 & 1600 & Spherical $^{b}$, Bipolar Ring $^{b}$ \\
\hline LMC-SMP 29 & - & 200 & - & \\
\hline SMC-SMP 1 & 37 & 41 & 500 & Spherical $^{b, c}$, Round $^{a}$ \\
\hline SMC-SMP 3 & 95 & 92 & $>2000$ & Bipolar Ring $^{b, c}$, Bipolar w/bipolar core ${ }^{a}$ \\
\hline SMC-SMP 22 & - & 200 & - & Bipolar Ring $^{b}$, Bipolar w/bipolar core ${ }^{a}$ \\
\hline
\end{tabular}

REFERENCES. - (aa): This paper. (bb): Dopita \& Meatheringham (1991a b);

Dopita et al. (1997); Vassiliadis et al. (1996, 1998a) (a): Stanghellini et al. (1999) (b): Vassiliadis et al. (1998b) (c): Dopita et al. (1996) (d): Shaw et al. (2001)

TABLE 8

LEVELS AND SUPERLEVELS FOR MODEL IONS

\begin{tabular}{|c|c|c|c|c|c|c|c|c|c|c|c|}
\hline Elemen & I & II & III & IV & $\mathrm{V}$ & VI & VII & VIII & IX & $\mathrm{X}$ & $\mathrm{XI}$ \\
\hline $\mathrm{H}$ & $20 / 30$ & $1 / 1$ & & & & & & & & & \\
\hline $\mathrm{He}$ & $40 / 45$ & $22 / 30$ & $1 / 1$ & & & & & & & & \\
\hline $\mathrm{C}$ & & & $30 / 54$ & $13 / 18$ & $1 / 1$ & & & & & & \\
\hline $\mathrm{N}$ & & & & $29 / 53$ & $13 / 21$ & $1 / 1$ & & & & & \\
\hline $\mathrm{O}$ & & & & $29 / 48$ & $41 / 78$ & $13 / 19$ & $1 / 1$ & & & & \\
\hline $\mathrm{Si}$ & & & & $22 / 33$ & $1 / 1$ & & & & & & \\
\hline $\mathrm{P}$ & & & & $36 / 178$ & $16 / 62$ & $1 / 1$ & & & & & \\
\hline $\mathrm{S}$ & & & & $51 / 142$ & $31 / 98$ & $28 / 58$ & $1 / 1$ & & & & \\
\hline $\mathrm{Fe}$ & & & & $51 / 294$ & $47 / 191$ & $44 / 433$ & $41 / 254$ & $53 / 324$ & $52 / 490$ & $43 / 210$ & $1 / 1$ \\
\hline
\end{tabular}

